\title{
Chitosan decorated copper nanoparticles as efficient catalyst for synthesis of novel quinoline derivatives
}

\author{
K.S. Alghamdi ${ }^{1}$, N.S.I. Ahmed ${ }^{2}$, D. Bakhotmah ${ }^{1}$ and M. Mokhtar ${ }^{1,3^{*}}$ \\ ${ }^{1}$ Chemistry Department, Faculty of Science, King Abdulaziz University, Jeddah 21589, Saudi Arabia \\ ${ }^{2}$ Department of Therapeutic Chemistry, Pharmaceutical and Drug Industries Research Division, National \\ Research Centre, El Buhouth St., Dokki, Cairo 12622, Egypt \\ ${ }^{3}$ Physical Chemistry Department, National Research Centre, El Buhouth St., Dokki, Cairo 12622, Egypt
}

*Corresponding author: Name: Mohamed Mokhtar, Email: mmoustsfa@ kau.edu.sa

Submitted/Received: Date 3-12-2018 Accepted: .........Date.........

\begin{abstract}
Chitosan decorated copper nanoparticles (CS/CuNPs) catalysts were synthesized via reduction methods utilizing green protocol. The CS/CuNPs hybrid catalysts were tested for the synthesis of quinoline derivatives utilizing one-pot multicomponent reaction (MCR) under ultrasonic irradiation. The best catalyst (CS/CuNPs) that provided good conversion reaction yield and high turnover frequency (TOF) was characterized using Fourier transform infrared (FTIR), Thermogravimetric analyses (TGA), X-ray diffraction (XRD), , scanning electron microscopy (SEM), transmission electron microscope (TEM) and X-ray photoelectron spectroscopy (XPS) techniques. Generalization of the scope of the proposed catalytic process was studied using different aldehydes. Excellent products yield and high TOF in even shorter reaction time ( $\sim \mathrm{min})$ was attained. Recyclability performance of the catalyst over five times re-use without detectable loss in product yield was recorded. The current method is green process utilizing environmentally benign catalyst and considered to be promising sustainable protocol for the synthesis of fine chemicals.
\end{abstract}

Keywords: Chitosan-copper NPs; Quinolone derivatives; Ultrasonic irradiation; One- pot synthesis; Greensustainable perspectives

\section{Introduction}

Quinolone and their derivatives have several biological activities, such as anti-malaria [1],anticancer[2,3] , antiinflammatory [4,5], anti-bacterial [5] , anti-asthmatic [6], anti-platelet activity [7] ,anti-hypertensive [8] .Therefore, several methods have been developed for the synthesis of quinolone derivatives utilizing various catalysts [9-12] . Recently, green and eco-friendly synthesis have attracted much attention. Numerous approaches have been established which improved green conditions to safer synthesis. ultrasonication is one of the auspicious green technology in the synthesis of organic compounds[13,14].

The use of nanoparticles (NPs) in catalysis is considered to be one of the most significant principles of green chemistry that is owing to a number of different reasons; the reaction time is short, diminishes generation of hazardous materials, economically visible as high yields produced with low cost [15]. Nanoparticles have been widely used as the catalyst support in organic transformation [16]. Our previous achievements in the synthesis of different organic synthons of important biological activities utilizing different nanosized solid heterogeneous catalysts under green protocol [17-22] revealed that exploring efficient, sustainable and green catalyst is crucial to achieve green sustainable perspectives. To attain our goal, natural bio-polymer supported heterogeneous nano-catalysts that have been utilized in recent years [23,24], was selected to be a catalyst support. One of the promising catalyst's support candidate is chitosan, which is produced by the N-deacetylation of chitin. It is considered to be the second most abundant natural polymer after cellulose [25]. Chitosan a chemically stable, 
non-toxic is an excellent candidate to be used as a support for copper and other transition metals due to its insolubility in organic solvents and the presence of functionalization of the free amine groups in the structure which is represented active sites for several of chemical modifications [26-28].

Nanoparticles have a special characteristic to aggregate and well clump together to form larger particles, thus nanoparticles lose their large surface area and other benefits [29]. Chitosan as a polymer-based stabilizes the nanoparticles to prevent their aggregation via coordination with metal nanoparticles through chelation mechanism, makes it a perfect support for metal nanoparticles[30] .

Gold, silver and transition metals nanoparticles such as palladium are available for the development of hybrid catalyst complexes and they can also be used in chemical transformation whereas a new glyoxal cross-linked chitosan Schiff base was prepared as a support material for palladium catalyzed Suzuki cross coupling reactions[31,32]. In addition, chitosan was used as support of copper nanoparticles as catalyst for the C-S coupling of thiophenol with aryl halides [33]. The synthesis of $\mathrm{Cu}$ nanoparticles using chitosan as both reducing and capping agent have been reported [34,35]. This single step method is considered to be costeffective, convenient and eco-friendly relative to other method of preparation [34] .

In the present work, chitosan decorated copper nanoparticles catalysts were synthesized through green methods [34,35] and its application as an efficient catalyst in multicomponent reaction to the synthesis of novel quinolone derivatives under ultrasonic irradiation were extensively studied. The CS/CuNPscatalyst is a promising efficient sustainable green catalyst for the synthesis of quinolone derivatives in satisfactory yield in short reaction time under ultrasonication conditions.

\section{Experimental Details}

\subsection{Materials}

Chitosan (molecular weight 100,000-300,000) (Acros Organics- Belgium). Sodium tripolyphosphate (Acros Organics- Belgium), copper (II) acetate monohydrate (Central Drug House CDH, New Delhi, India), dimedone, ethyl cyanoacetate, and ammonium acetate (Techno Pharm Chem, New Delhi, India). 4-chlorobenzaldehyde, 4bromo benzaldehyde, 4-florobenzaldhyde, piperonal(1,3-benzodioxole-5-carbaldehyde), salicylaldehyde (2hydroxybenzaldehyde, thiophen -3-aldehyde,4-dimethylaminobenzaldehyde, 2-methoxybenzaldhyde,3,4dimethoxy benzaldehyde, m-nitro benzaldehyde (Merck KGaA, Darmstadt, Germany), absolute ethanol and acetone (Fisher scientific, Leicestershire, U.K).

\subsection{Materials characterization}

The reactions were monitored by TLC and all yields refer to isolated products. Melting points were obtained by the Barnstead international 1002 melting point apparatus. IR spectra of the catalyst and products were recorded for the compounds in PerkinElmer spectrum 100 FT-IR spectrophotometer. ${ }^{1} \mathrm{H}$ NMR and ${ }^{13} \mathrm{C}$ spectra of products were recorded on Burker WM 400 and $850 \mathrm{MHz}$ spectrometer using TMS (0.00 ppm). Chemical shifts $(\delta)$ are given in ppm relative to the signal for TMS as standard, and coupling constants in Hz. ThermoMicrobalance TG 209 F3 (NETZSCH) was used to perform thermal gravimetric analysis for the prepared samples. The sample was first heated form room temperature up to $150^{\circ} \mathrm{C}, 5^{\circ} \mathrm{C} / \mathrm{min}$, and kept half an hour at $150^{\circ} \mathrm{C}$. Then the sample temperature was raised up to $550^{\circ} \mathrm{C}$, afterwards cooled down to $40^{\circ} \mathrm{C}$ at the same rate of $10^{\circ} \mathrm{C} / \mathrm{min}$. Mixture of $20 \mathrm{ml} / \mathrm{min}$ of dry air and $10 \mathrm{ml} / \mathrm{min}$ of helium was passed through during these processes. PXRD patterns catalyst sample were analyzed using a Powder XRD diffractometer (Model Equinox 1000 - INEL (France) with Co $\mathrm{K}_{\alpha}(\lambda=1.7890 \AA \AA \mathrm{\AA})$ radiation at $30 \mathrm{kV}$ and $30 \mathrm{~mA}$. Catalyst morphology was investigated by means of field emission scanning electron microscopy (FEG-SEM, Quanta FEG450, FEI, the Netherlands) using an ETD Everhart Thornley detector (High Vacuum mode), a solid-state backscattering electron detector (VCD)and EDS detector (XFLASH6-30, Brucker) for elemental analysis. XPS measurements were carried out in an ultra-high vacuum multi- technique surface analysis system (SPECS GmbH, Germany) operating at a base pressure range of 1010 bar. HRTEM samples were prepared by sonication of the suspended powder in ethanol. A single drop of the sonicated suspension was deposited on TEM carbon grid 200 mesh and leaved for total evaporation at room temperature. Then the grid was mounted on a TEM single tilt holder, the residual solvent was removed by plasma cleaning process. The reactions that carried out by U.S irradiation was 
done using Daihan (Wiseclean, D-40 MHz) ultrasonic bath. Microanalysis was performed by Perkin Elmer elemental analyzer at the Faculty of Science, King Abdul Aziz University.

\subsection{Method}

\subsubsection{Synthesis of Chitosan decorated copper nanoparticles Catalyst}

The synthesis of Chitosan decorated copper nanoparticles was carried out according to two methods:

Firstly: (CS/CuNPs) have been prepared via one-step synthesis green protocol. In a typical method, $0.75 \mathrm{~g}$ chitosan dissolving in $100 \mathrm{ml} 0.1 \%$ acetic acid (in distilled water) then $50 \mathrm{ml}$ of the solution and $25 \mathrm{ml}$ of 0.05 $\mathrm{M}$ copper solution were delivered under stirring at $70^{\circ} \mathrm{C}$ for $9 \mathrm{~h}$ till the reaction was completed. the colloid was centrifuged for $10 \mathrm{~min}$. to separate particles from suspension then washed with acetone $(90 \%$, v/v) and the centrifugation was repeated three times to remove unreacted reagents. The particles were dried under vacuum at the room temperature overnight and stored [34].

Secondly: (CS/CuNPs/TPP) were prepared based on the ionotropic gelation between chitosan and sodium tripolyphosphate (TTP). Chitosan acted as a reducing/stabilizing agent. TPP was dissolved in water to a concentration of $0.25 \%$. Under magnetic stirring at room temperature, $33 \mathrm{ml}$ of TTP solution was added into 50 $\mathrm{ml}$ of chitosan solution $0.75 \%$ (in dil. acetic acid $0.1 \%$ ) and the mixture was stirred for 15min.Chitosan nanoparticles loaded $\mathrm{Cu}^{2+}$ were obtained by adding metal ion solutions $16 \mathrm{ml} 0.05 \mathrm{M}$ into the chitosan nanosuspensions and heated to $70{ }^{\circ} \mathrm{C}$ using a water bath, after a blue color appeared, stirring continued for another $90 \mathrm{~min}$. before removing the heater. The resulting solution was cooled to room temperature for characterization [35] .

\subsubsection{Synthesis of polyhydroquinolines in the presence of CS/CuNPs}

A mixture of dimedone (3.6 mmol, $0.5 \mathrm{gm})$, aromatic aldehyde $(3.6 \mathrm{mmol}, 0.5 \mathrm{gm})$, ethylcyanoacetoacetate (3.6 mmol, $0.38 \mathrm{ml})$, ammoniumacetate $(28.8 \mathrm{mmol}, 2.219 \mathrm{gm})$ and catalytic amounts of Cu-chitosan NPs $(0.1 \mathrm{gm})$ in $10 \mathrm{ml}$ absolute ethanol irradiate with ultrasonic waves at $80^{\circ} \mathrm{C}$. After completion of the reaction (monitored by TLC, petroleum ether: EtOAc, 1:2), the reaction mixture was filtered to separate the catalyst, then cooled at room temperature and the solid product obtained and was filtered off, dried and recrystallized from ethanol.

\subsubsection{Physical and spectroscopic data of product compounds}

Ethyl-2-amino-4-(4-chlorophenyl)-7,7-dimethyl-5-oxo-1,4,5,6,7,8hexahydroquinoline-3-carboxylate (2a) Off - white crystals $\left(1.09 \mathrm{gm}, 82.47 \%\right.$ yield); m.p $173{ }^{\circ} \mathrm{C}$. FTIR; 3478,3328, $3200\left(-\mathrm{NH}, \mathrm{NH}_{2}\right) ; 1686,1655$ $(2 \mathrm{C}=\mathrm{O})$; and $1621 \mathrm{~cm}^{-1}(\mathrm{C}=\mathrm{C}) .{ }^{1} \mathrm{H}$ NMR $\left(400 \mathrm{MHz}, \mathrm{CDCl}_{3}\right): \delta_{\mathrm{H}}=0.96,1.09\left(6 \mathrm{H}, 2 \mathrm{~s}, 2 \mathrm{CH}_{3}\right) ; 1.14(3 \mathrm{H}, \mathrm{t}$, $\left.\mathrm{CH}_{2} \mathrm{CH}_{3}, J=7.2 \mathrm{~Hz}\right) ; 1.84(1 \mathrm{H}$, br.s, $-\mathrm{NH}) ; 2.17\left(2 \mathrm{H}, \mathrm{dd}, \mathrm{C}_{8}-\mathrm{H}, J=14 \mathrm{~Hz}\right) ; 2.41\left(2 \mathrm{H}, \mathrm{s}, \mathrm{C}_{6}-\mathrm{H}\right) ; 4.03(2 \mathrm{H}, \mathrm{q},-$ $\left.\mathrm{CH}_{2} \mathrm{CH}_{3}, J=7.2 \mathrm{~Hz}\right) ; 4.66\left(1 \mathrm{H}, \mathrm{s}, \mathrm{C}_{4}-\mathrm{H}\right) ; 6.21\left(2 \mathrm{H}\right.$, br.s, $\left.\mathrm{NH}_{2}\right)$ and $7.15,7.21(4 \mathrm{H}, 2 \mathrm{~d}, \mathrm{Ar}-\mathrm{H}) .{ }^{13} \mathrm{C}$ NMR $\left(\mathrm{CDCl}_{3}\right): \delta_{\mathrm{C}}=14.22\left(\mathrm{CH}_{2} \mathrm{CH}_{3}\right) ; 27.36,29.09\left(2 \mathrm{CH}_{3}\right) ; 32.24\left(\mathrm{C}_{7}\right) ; 33.48\left(\mathrm{C}_{4}\right) ; 40.66\left(\mathrm{C}_{8}\right) ; 50.69\left(\mathrm{C}_{6}\right) ; 59.77$ $\left(\underline{\mathrm{CH}_{2}} \mathrm{CH}_{3}\right) ; 80.30\left(\mathrm{C}_{3}\right) ; 116.40\left(\mathrm{C}_{4 \mathrm{a}}\right) ; 127.91\left(\mathrm{C}_{3}{ }^{\prime}, \mathrm{C}_{5}{ }^{\prime}\right) ; 129.63\left(\mathrm{C}_{2}{ }^{\prime}, \mathrm{C}_{6}{ }^{\prime}\right) ; 131.63\left(\mathrm{C}_{4}{ }^{\prime}\right) ; 144.46\left(\mathrm{C}_{1}{ }^{\prime}\right) ; 158.37$ $\left(\mathrm{C}_{8 \mathrm{a}}\right) ; 161.54,196.43(2 \mathrm{C}=\mathrm{O})$; and $168.95\left(\mathrm{C}_{2}\right)$. Anal. Calcd. for $\mathrm{C}_{20} \mathrm{H}_{23} \mathrm{ClN}_{2} \mathrm{O}_{3}$ (374.86): $\mathrm{C}, 64.02 ; \mathrm{H}, 6.61 ; \mathrm{N}$, 7.47; O, 12.80. Found : C, 64.42; H, 6.43; N, 7.01; O, 12.58 .

Ethyl-2-amino-4-(4-bromophenyl)-7,7-dimethyl-5-oxo-1,4,5,6,7,8hexahydroquinoline-3-carboxylate ( $\mathbf{2}_{\mathrm{b}}$ ) Semisolid Off - white (1.41 gm, $93.49 \%$ yield). FTIR; 3473,3331,3204 (-NH, NH2); 1686,1654 (2C=O ); and $1620 \mathrm{~cm}-1(\mathrm{C}=\mathrm{C}) .1 \mathrm{H}$ NMR $(850 \mathrm{MHz}, \mathrm{CDCl} 3): \delta \mathrm{H}=0.96,1.09(6 \mathrm{H}, 2 \mathrm{~s}, 2 \mathrm{CH} 3) ; 1.15(3 \mathrm{H}, \mathrm{t},-\mathrm{CH} 2 \mathrm{CH} 3$, $\mathrm{J}=7.56 \mathrm{~Hz}) ; 2.08(1 \mathrm{H}, \mathrm{br} . \mathrm{s},-\mathrm{NH}) ; 2.18(2 \mathrm{H}, \mathrm{dd}, \mathrm{C} 8-\mathrm{H}, \mathrm{J}=14 \mathrm{~Hz}) ; 2.42(2 \mathrm{H}$, d.d , C6-H , J= $17 \mathrm{HZ}) ; 4.03$ (2H, q, $-\mathrm{CH} 2 \mathrm{CH} 3, \mathrm{~J}=7.56 \mathrm{~Hz}) ; 4.65(1 \mathrm{H}, \mathrm{s}, \mathrm{C} 4-\mathrm{H}) ; 6.20(2 \mathrm{H}$, br.s, NH2) and 7.13, $7.33(4 \mathrm{H}, 2 \mathrm{~d}, \mathrm{Ar}-\mathrm{H}) .13 \mathrm{C}$ NMR (CDCl3): $\delta \mathrm{C}=14.21$ (CH2CH3); 27.36, 29.10 (2CH3); 32.25 (C7); 33.44 (C4); 40.63 (C8); 50.65 (C6); 59.79

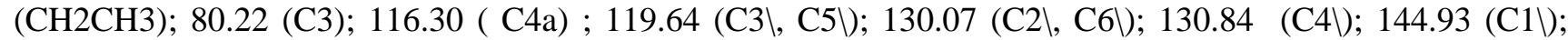
158.31 (C8a); $161.55,196.46$ (2 C=O); and 168.94 (C2) . Anal. Calcd. for C20H23BrN2O3 (419.31): C, 57.24; $\mathrm{H}, 4.48 ; \mathrm{N}, 6.68 ; \mathrm{O}, 11.45$. Found : C, 57.40; H, 4.33; N, 6.43; O, 11.30 . 
Off - white crystals $\left(1.08\right.$ gm, $84.65 \%$ yield); m.p $154{ }^{\circ} \mathrm{C}$. FTIR; 3398,3285, $3200\left(-\mathrm{NH}, \mathrm{NH}_{2}\right) ; 1689,1652$ $(2 \mathrm{C}=\mathrm{O})$; and $1601 \mathrm{~cm}^{-1}(\mathrm{C}=\mathrm{C}) .{ }^{1} \mathrm{H}$ NMR $\left(850 \mathrm{MHz}, \mathrm{CDCl}_{3}\right): \delta_{\mathrm{H}}=0.96,1.09\left(6 \mathrm{H}, 2 \mathrm{~s}, 2 \mathrm{CH}_{3}\right) ; 1.14(3 \mathrm{H}$, t, $\left.\mathrm{CH}_{2} \mathrm{CH}_{3}, J=7.65 \mathrm{~Hz}\right) ; 2.06(1 \mathrm{H}$, br.s, $-\mathrm{NH}) ; 2.17\left(2 \mathrm{H}, \mathrm{dd}, \mathrm{C}_{8}-\mathrm{H}, J=16.15 \mathrm{~Hz}\right) ; 2.41$ ( 2H, d.d , $\mathrm{C}_{6}-\mathrm{H}, J=17.83$ $\mathrm{HZ}) ; 4.03\left(2 \mathrm{H}, \mathrm{q},-\mathrm{CH}_{2} \mathrm{CH}_{3}, J=6.8 \mathrm{~Hz}\right) ; 4.67\left(1 \mathrm{H}, \mathrm{s}, \mathrm{C}_{4}-\mathrm{H}\right) ; 6.26\left(2 \mathrm{H}\right.$, br.s, $\left.\mathrm{NH}_{2}\right)$ and 6.87, $7.23(4 \mathrm{H}, 2 \mathrm{~d}, \mathrm{Ar}-$ H). ${ }^{13} \mathrm{C}$ NMR $\left(\mathrm{CDCl}_{3}\right): \delta_{\mathrm{C}}=14.21\left(\mathrm{CH}_{2} \mathrm{CH}_{3}\right) ; 27.33,29.08\left(2 \mathrm{CH}_{3}\right) ; 32.23\left(\mathrm{C}_{7}\right) ; 33.24\left(\mathrm{C}_{4}\right) ; 40.62\left(\mathrm{C}_{8}\right) ; 50.68$ $\left(\mathrm{C}_{6}\right) ; 59.71\left(\underline{\mathrm{CH}}_{2} \mathrm{CH}_{3}\right) ; 80.53\left(\mathrm{C}_{3}\right) ; 114.54\left(\mathrm{C}_{4 \mathrm{a}}\right) ; 116.61\left(\mathrm{C}_{3}{ }^{\prime}, \mathrm{C}_{5}^{\prime}\right) ; 129.65\left(\mathrm{C}_{2}^{\prime}, \mathrm{C}_{6}^{\prime}\right) ; 141.65\left(\mathrm{C}_{4}^{\prime}\right) ; 158.32\left(\mathrm{C}_{1}^{\prime}\right)$; $160.65\left(\mathrm{C}_{8 \mathrm{a}}\right) ; 161.42,196.53(2 \mathrm{C}=\mathrm{O})$; and $169.03\left(\mathrm{C}_{2}\right)$. Anal. Calcd. for $\mathrm{C}_{20} \mathrm{H}_{23} \mathrm{FN}_{2} \mathrm{O}_{3}(358.41)$ : $\mathrm{C}, 66.96 ; \mathrm{H}$, $6.42 ; \mathrm{N}, 7.81 ; \mathrm{O}, 13.39$. Found : C, 66.98; H, 6.27; N, 7.40; O, 13.11.

Ethyl-2-amino-4-(3-nitrophenyl)-7,7-dimethyl-5-oxo-1,4,5,6,7,8hexahydroquinoline-3-carboxylate $\left(2_{\mathrm{d}}\right)$ Dark green crystals (1.04 gm, $75.89 \%$ yield); m.p $147{ }^{\circ} \mathrm{C}$. FTIR; 3449,3332, $3200\left(-\mathrm{NH}, \mathrm{NH}_{2}\right) ; 1371\left(\mathrm{NO}_{2}\right)$; $1686,1657(2 \mathrm{C}=\mathrm{O})$; and $1621 \mathrm{~cm}^{-1}(\mathrm{C}=\mathrm{C}) .{ }^{1} \mathrm{H}$ NMR $\left(850 \mathrm{MHz}, \mathrm{CDCl}_{3}\right): \delta_{\mathrm{H}}=0.96,1.09\left(6 \mathrm{H}, 2 \mathrm{~s}, 2 \mathrm{CH}_{3}\right) ; 1.14$ $\left(3 \mathrm{H}, \mathrm{t},-\mathrm{CH}_{2} \mathrm{CH}_{3}, J=7.65 \mathrm{~Hz}\right) ; 2.10(1 \mathrm{H}$,br.s, $-\mathrm{NH}) ; 2.17\left(2 \mathrm{H}, \mathrm{dd}, \mathrm{C}_{8}-\mathrm{H}, J=16.15 \mathrm{~Hz}\right) ; 2.47\left(2 \mathrm{H}, \mathrm{s}, \mathrm{C}_{6}-\mathrm{H}\right)$; $4.03\left(2 \mathrm{H}, \mathrm{q},-\mathrm{CH}_{2} \mathrm{CH}_{3}, J=6.8 \mathrm{~Hz}\right) ; 4.79\left(1 \mathrm{H}, \mathrm{s}, \mathrm{C}_{4}-\mathrm{H}\right) ; 6.31\left(2 \mathrm{H}\right.$, br.s, $\left.\mathrm{NH}_{2}\right)$ and $7.37\left(1 \mathrm{H}, \mathrm{d} . \mathrm{d}, \mathrm{C}_{5}-\mathrm{H}, J=7.65\right.$, $J=8.5 \mathrm{~Hz}$ of $\mathrm{Ar}) ; 7.64\left(1 \mathrm{H}, \mathrm{d}, \mathrm{C}_{4}-\mathrm{H}, J=7.65\right.$ of $\left.\mathrm{Ar}\right) ; 7.98\left(1 \mathrm{H}, \mathrm{d}, \mathrm{C}_{6}-\mathrm{H}, J=8.5 \mathrm{~Hz}\right.$ of $\left.\mathrm{Ar}\right) ; 8.09\left(1 \mathrm{H}, \mathrm{S}, \mathrm{C}_{2}-\mathrm{H}\right.$ of $\mathrm{Ar}) .\left({ }^{13} \mathrm{C}\right.$ NMR $\left(\mathrm{CDCl}_{3}\right): \delta_{\mathrm{C}}=14.19\left(\mathrm{CH}_{2} \underline{\mathrm{CH}}_{3}\right) ; 27.37,29.06\left(2 \mathrm{CH}_{3}\right) ; 32.31\left(\mathrm{C}_{7}\right) ; 34.15\left(\mathrm{C}_{4}\right) ; 40.61\left(\mathrm{C}_{8}\right) ; 50.58$ $\left(\mathrm{C}_{6}\right) ; 59.91\left(\underline{\mathrm{CH}}_{2} \mathrm{CH}_{3}\right) ; 79.51\left(\mathrm{C}_{3}\right) ; 115.56\left(\mathrm{C}_{4 \mathrm{a}}\right) ; 121.35\left(\mathrm{C}_{4}{ }^{\prime}, \mathrm{C}_{6}{ }^{\prime}\right) ; 123.16\left(\mathrm{C}_{5}^{\prime}\right) ; 128.51\left(\mathrm{C}_{2}^{\prime}\right) ; 134.91\left(\mathrm{C}_{3}{ }^{\prime}\right)$; $148.14\left(\mathrm{C}_{3}^{\prime}\right) ; 158.36\left(\mathrm{C}_{8 \mathrm{a}}\right) ; 162.15,196.43(2 \mathrm{C}=\mathrm{O})$; and $168.66\left(\mathrm{C}_{2}\right)$. Anal. Calcd. for $\mathrm{C}_{20} \mathrm{H}_{23} \mathrm{~N}_{3} \mathrm{O}_{5}(385.41)$ : $\mathrm{C}$, 62.27; H, 5.97; N, 10.89; O, 20.76. Found : C, 62.48; H, 5.53; N, 10.45; O, 12.63.

Ethyl-2-amino-7,7-dimethyl-5-oxo-4-(thiophen-3-yl)-1,4,5,6,7,8-hexahydroquinoline-3-carboxylate $\left(2_{\mathrm{e}}\right)$ Drak brown powder (1.22 gm, 98.53\% yield); m.p $123{ }^{\circ} \mathrm{C}$. FTIR; 3427,3310, $3208\left(-\mathrm{NH}, \mathrm{NH}_{2}\right) ; 1688,1654$ $(2 \mathrm{C}=\mathrm{O})$; and $1636 \mathrm{~cm}^{-1}(\mathrm{C}=\mathrm{C}) .{ }^{1} \mathrm{H}$ NMR $\left(850 \mathrm{MHz}, \mathrm{CDCl}_{3}\right): \delta_{\mathrm{H}}=1.01,1.12\left(6 \mathrm{H}, 2 \mathrm{~s}, 2 \mathrm{CH}_{3}\right) ; 1.20(3 \mathrm{H}, \mathrm{t},-$ $\left.\mathrm{CH}_{2} \mathrm{CH}_{3}, J=6.8 \mathrm{~Hz}\right) ; 2.13(1 \mathrm{H}, \mathrm{br} . \mathrm{s},-\mathrm{NH}) ; 2.26\left(2 \mathrm{H}, \mathrm{dd}, \mathrm{C}_{8}-\mathrm{H}, J=16.15 \mathrm{~Hz}\right) ; 2.42\left(2 \mathrm{H}, \mathrm{s}, \mathrm{C}_{6}-\mathrm{H}\right) ; 4.10(2 \mathrm{H}, \mathrm{q}$, $\left.-\mathrm{CH}_{2} \mathrm{CH}_{3}, J=7.65 \mathrm{~Hz}\right) ; 4.89\left(1 \mathrm{H}, \mathrm{s}, \mathrm{C}_{4}-\mathrm{H}\right) ; 6.19\left(2 \mathrm{H}\right.$, br.s, $\left.\mathrm{NH}_{2}\right)$ and $6.95\left(1 \mathrm{H}, \mathrm{d}, \mathrm{C}_{5}-\mathrm{H}\right.$ of thiophene $), 7.10$ $\left(1 \mathrm{H}, \mathrm{S}, \mathrm{C}_{2}-\mathrm{H}\right.$ of thiophene $), 7.12\left(1 \mathrm{H}, \mathrm{m}, \mathrm{C}_{4}-\mathrm{H}\right.$ of thiophene) $\quad{ }^{13} \mathrm{C}$ NMR $\left(\mathrm{CDCl}_{3}\right): \delta_{\mathrm{C}}=14.18\left(\mathrm{CH}_{2} \underline{\mathrm{CH}}_{3}\right)$; 27.44, $28.65\left(2 \mathrm{CH}_{3}\right) ; 32.24\left(\mathrm{C}_{7}\right) ; 40.67\left(\mathrm{C}_{8}\right) ; 41.21\left(\mathrm{C}_{4}\right) ; 50.74\left(\mathrm{C}_{6}\right) ; 59.73\left(\mathrm{CH}_{2} \mathrm{CH}_{3}\right) ; 80.38\left(\mathrm{C}_{3}\right) ; 116.61\left(\mathrm{C}_{4 \mathrm{a}}\right)$ ; $120.92\left(\mathrm{C}_{3}{ }^{\prime}\right) ; 124.55\left(\mathrm{C}_{2}{ }^{\prime}\right) ; 127.71\left(\mathrm{C}_{4}{ }^{\prime}\right) ; 147.76\left(\mathrm{C}_{1}{ }^{\prime}\right) ; 158.73\left(\mathrm{C}_{8 \mathrm{a}}\right) ; 162.03,197.34(2 \mathrm{C}=\mathrm{O})$; and $169.35\left(\mathrm{C}_{2}\right)$ . Anal. Calcd. for $\mathrm{C}_{18} \mathrm{H}_{22} \mathrm{~N}_{2} \mathrm{O}_{3} \mathrm{~S}(346.44)$ : C, 62.35; H, 6.35;N, 8.08; O, 13.85; S,9.24 . Found : C, 62.22; H, $6.16 ; \mathrm{N}, 8.01 ; \mathrm{O}, 13.46 ; \mathrm{S}, 9.17$.

\section{Ethyl-2-amino-4-(3,4-dimethoxyphenyl)-7,7-dimethyl-5-oxo-1,4,5,6,7,8-hexahydro-quinoline-3- carboxylate $\left(\mathbf{2}_{\mathrm{f}}\right)$}

Off - white powder (1.17 gm, $81.59 \%$ yield); m.p $128{ }^{\circ} \mathrm{C}$. FTIR; 3429,3314, $3200\left(-\mathrm{NH}, \mathrm{NH}_{2}\right) ; 1687,1663$ $(2 \mathrm{C}=\mathrm{O})$; and $1588 \mathrm{~cm}^{-1}(\mathrm{C}=\mathrm{C}) .{ }^{1} \mathrm{H}$ NMR $\left(850 \mathrm{MHz} \mathrm{CDCl}_{3}\right): \delta_{\mathrm{H}}=0.98,1.10\left(6 \mathrm{H}, 2 \mathrm{~s}, 2 \mathrm{CH}_{3}\right) ; 1.19(3 \mathrm{H}$, t, $\left.\mathrm{CH}_{2} \mathrm{CH}_{3}, J=6.8 \mathrm{~Hz}\right) ; 2.13(1 \mathrm{H}$, br.s, $-\mathrm{NH}) ; 2.19\left(2 \mathrm{H}, \mathrm{dd}, \mathrm{C}_{8}-\mathrm{H}, J=16.9 \mathrm{~Hz}\right) ; 2.41\left(2 \mathrm{H}\right.$, d.d , $\mathrm{C}_{6}-\mathrm{H}, J=17.85 \mathrm{~Hz}$ ); 3.96,3.97(6H,2S,2-OCH $) ; 4.38\left(2 \mathrm{H}, \mathrm{q},-\mathrm{CH}_{2} \mathrm{CH}_{3}, J=7.65 \mathrm{~Hz}\right) ; 4.65\left(1 \mathrm{H}, \mathrm{s}, \mathrm{C}_{4}-\mathrm{H}\right) ; 6.69\left(2 \mathrm{H}, \mathrm{br} . \mathrm{s}, \mathrm{NH}_{2}\right)$ and 7.74,7.80,8.16 (3H, d.d, s, Ar-H). ${ }^{13} \mathrm{C}$ NMR $\left(\mathrm{CDCl}_{3}\right): \delta_{\mathrm{C}}=14.22\left(\mathrm{CH}_{2} \underline{\mathrm{CH}}_{3}\right) ; 27.30,29.27\left(2 \mathrm{CH}_{3}\right) ; 32.24\left(\mathrm{C}_{7}\right)$; $33.29\left(\mathrm{C}_{4}\right) ; 40.67\left(\mathrm{C}_{8}\right) ; 50.81\left(\mathrm{C}_{6}\right) ; 55.65,56.07\left(2-\mathrm{OCH}_{3}\right) ; 59.69\left(\underline{\mathrm{CH}}_{2} \mathrm{CH}_{3}\right) ; 80.92\left(\mathrm{C}_{3}\right) ; 110.995\left(\mathrm{C}_{4 a}\right) 111.66$ $\left(\mathrm{C}_{5}^{\prime}\right)$; $112.02\left(\mathrm{C}_{2}{ }^{\prime}\right) ; 138.71 \quad\left(\mathrm{C}_{1}^{\prime}\right) ; 147.15\left(\mathrm{C}_{4}{ }^{\prime}\right) ; 148.36\left(\mathrm{C}_{3}{ }^{\prime}\right) ; ; 149.29\left(\mathrm{C}_{8 \mathrm{a}}\right) ; 161.26,196.62(2 \mathrm{C}=\mathrm{O})$; and 163.13( $\left.\mathrm{C}_{2}\right)$. Anal. Calcd. for $\mathrm{C}_{22} \mathrm{H}_{28} \mathrm{~N}_{2} \mathrm{O}_{5}$ (400.47): C, 65.92; H, 6.99; N, 6.99; O, 19.98. Found: C, 65.99; H, $6.61 ; \mathrm{N}, 6,36 ; \mathrm{O}, 19.44$.

Ethyl-2-amino-4-(benzo[d][1,3]dioxol-5-yl)-7,7-dimethyl-5-oxo-1,4,5,6,7,8-hexahydrocarboxylate $\left(2_{\mathrm{g}}\right)$

Off - white crystals $\left(1.20\right.$ gm, 87.36\% yield); m.p $133{ }^{\circ} \mathrm{C}$. FTIR; 3437, $3204\left(-\mathrm{NH}, \mathrm{NH}_{2}\right) ; 1688,1653(2 \mathrm{C}=\mathrm{O})$; and $1606 \mathrm{~cm}^{-1}(\mathrm{C}=\mathrm{C}) .{ }^{1} \mathrm{H}$ NMR $\left(850 \mathrm{MHz}, \mathrm{CDCl}_{3}\right): \delta_{\mathrm{H}}=0.99,1.09\left(6 \mathrm{H}, 2 \mathrm{~s}, 2 \mathrm{CH}_{3}\right) ; 1.18\left(3 \mathrm{H}, \mathrm{t},-\mathrm{CH}_{2} \mathrm{CH}_{3}\right.$, $J=7.65 \mathrm{~Hz}) ; 2.08(1 \mathrm{H}$, br.s, $-\mathrm{NH}) ; 2.19\left(2 \mathrm{H}, \mathrm{dd}, \mathrm{C}_{8}-\mathrm{H}, J=16.15 \mathrm{~Hz}\right) ; 2.41\left(2 \mathrm{H}, \mathrm{s}, \mathrm{C}_{6}-\mathrm{H}\right) ; 4.36(2 \mathrm{H}, \mathrm{q},-$ $\left.\mathrm{CH}_{2} \mathrm{CH}_{3}, J=6.8 \mathrm{~Hz}\right) ; 4.62\left(1 \mathrm{H}, \mathrm{s}, \mathrm{C}_{4}-\mathrm{H}\right) ; 5.87\left(2 \mathrm{H}, 2 \mathrm{~d},-\mathrm{O}-\mathrm{CH}_{2}-\mathrm{O}\right) ; 6.08\left(2 \mathrm{H}\right.$, br.s, $\left.\mathrm{NH}_{2}\right)$ and 6.65,6.74,6.90(3H, d,d.d,d, Ar-H). ${ }^{13} \mathrm{C}$ NMR $\left(\mathrm{CDCl}_{3}\right): \delta_{\mathrm{C}}=14.28\left(\mathrm{CH}_{2} \underline{\mathrm{CH}}_{3}\right) ; 27.52,29.04\left(2 \mathrm{CH}_{3}\right) ; 32.25\left(\mathrm{C}_{7}\right) ; 33.29\left(\mathrm{C}_{4}\right) ; 40.64$ $\left(\mathrm{C}_{8}\right) ; 51.23\left(\mathrm{C}_{6}\right) ; 59.72\left(\mathrm{CH}_{2} \mathrm{CH}_{3}\right) ; 80.88\left(\mathrm{C}_{3}\right) ; 100.68\left(-\mathrm{O}-\mathrm{CH}_{3}-\mathrm{O}\right) ; 107.59\left(\mathrm{C}_{4 a}\right) ; 109.21\left(\mathrm{C}_{5}{ }^{\prime}\right) ; 116.82\left(\mathrm{C}_{2}{ }^{\prime}\right)$; $121.34\left(\mathrm{C}_{6}{ }^{\prime}\right) ; 139.98\left(\mathrm{C}_{1}{ }^{\prime}\right) ; 145.65\left(\mathrm{C}_{4}{ }^{\prime}\right) ; 147.07\left(\mathrm{C}_{3}{ }^{\prime}\right) ; 158.20\left(\mathrm{C}_{8 \mathrm{a}}\right) ; 162.99,196.56(2 \mathrm{C}=\mathrm{O})$; and $169.11\left(\mathrm{C}_{2}\right)$. Anal. Calcd. for $\mathrm{C}_{21} \mathrm{H}_{24} \mathrm{~N}_{2} \mathrm{O}_{5}$ (384.17): C, 65.59; H, 6.25; N, 7.29; O, 20.82. Found: C, 65.92; H, 6.13; N, 7.01; O, 20.54 . 
Ethyl-2-amino-4-(2-dimethylamino(phenyl)-7,7-dimethyl-5-oxo-1,4,5,6,7,8 hexahydroquinol ine-3carboxylate $(2 \mathrm{~h})$

Light yellow crystals (1.04 gm, $75.95 \%$ yield); m.p $115{ }^{\circ} \mathrm{C}$. FTIR; 3196,2932, 3200 (-NH, NH NH $_{2} ; 1702,1610$ $(2 \mathrm{C}=\mathrm{O})$; and $1561 \mathrm{~cm}^{-1}(\mathrm{C}=\mathrm{C}) .{ }^{1} \mathrm{H}$ NMR $\left(850 \mathrm{MHz}, \mathrm{CDCl}_{3}\right): \delta_{\mathrm{H}}=1.08\left(6 \mathrm{H}, 2 \mathrm{~s}, 2 \mathrm{CH}_{3}\right) ; 1.38\left(3 \mathrm{H}, \mathrm{t},-\mathrm{CH}_{2} \underline{\mathrm{CH}}_{3}\right.$, $J=6.8 \mathrm{~Hz}) ; 1.61(1 \mathrm{H}, \mathrm{br} . \mathrm{s},-\mathrm{NH}) ; 2.14\left(2 \mathrm{H}, \mathrm{dd}, \mathrm{C}_{8}-\mathrm{H}, J=14 \mathrm{~Hz}\right) ; 2.26\left(2 \mathrm{H}, \mathrm{s}, \mathrm{C}_{6}-\mathrm{H}\right) ; 3.09\left(6 \mathrm{H}, \mathrm{s}, \mathrm{N}(\mathrm{CH} 3)_{2}\right) ; 4.34$ $\left(2 \mathrm{H}, \mathrm{q},-\mathrm{CH}_{2} \mathrm{CH}_{3}, J=7.65 \mathrm{~Hz}\right) ; 4.98\left(1 \mathrm{H}, \mathrm{s}, \mathrm{C}_{4}-\mathrm{H}\right) ; 6.64\left(2 \mathrm{H}\right.$, br.s, $\left.\mathrm{NH}_{2}\right)$ and $6.70,7.94(4 \mathrm{H}, 2 \mathrm{~d}, \mathrm{Ar}-\mathrm{H}) .{ }^{13} \mathrm{C}$ NMR $\left(\mathrm{CDCl}_{3}\right): \delta_{\mathrm{C}}=14.30\left(\mathrm{CH}_{2} \underline{\mathrm{CH}}_{3}\right) ; 27.38,28.34\left(2 \mathrm{CH}_{3}\right) ; 32.90\left(\mathrm{C}_{7}\right) ; 39.98\left(\mathrm{C}_{4}\right) ; 40.03\left(\mathrm{C}_{8}\right) ; 41.40,42.64(\mathrm{~N}-$ $\left.(\underline{\mathrm{CH}} 3)_{2}\right), 50.72\left(\mathrm{C}_{6}\right) ; 61.58\left(\underline{\mathrm{CH}}_{2} \mathrm{CH}_{3}\right) ; 94.06\left(\mathrm{C}_{3}\right) ; 110.09\left(\mathrm{C}_{4 \mathrm{a}}\right) ; 111.50,112.67\left(\mathrm{C}_{2}{ }^{\prime}, \mathrm{C}_{6}^{\prime}\right) ; 117.60,119.81\left(\mathrm{C}_{3}{ }^{\prime}\right.$, $\left.\mathrm{C}_{5}{ }^{\prime}\right) ; 134.07\left(\mathrm{C}_{1}{ }^{1}\right) ; 136.07\left(\mathrm{C}_{1}{ }^{\prime}\right) ; 154.58\left(\mathrm{C}_{8 \mathrm{a}}\right) ; 163.15,197.59(2 \mathrm{C}=\mathrm{O})$; and $164.32\left(\mathrm{C}_{2}\right)$. Anal. Calcd. for $\mathrm{C}_{22} \mathrm{H}_{29} \mathrm{~N}_{3} \mathrm{O}_{3}$ (383.48): C, 68.84; H, 7.56; N, 10.95; O, 12.52. Found: C, 68.92; H, 7.32; N, 10.61; O, 12.17.

Ethyl-2-amino-4-(2-methoxyphenyl)-7,7-dimethyl-5-oxo-1,4,5,6,7,8hexahydroquinoline-3-carboxylate $(2 \mathrm{i})$

Dark green crystals (0.96 gm, 71.99\% yield); m.p $185{ }^{\circ} \mathrm{C}$. FTIR; 3421,3309, $3200\left(-\mathrm{NH}, \mathrm{NH}_{2}\right) ; 1685,1649$ $(2 \mathrm{C}=\mathrm{O})$; and $1613 \mathrm{~cm}^{-1}(\mathrm{C}=\mathrm{C}) .{ }^{1} \mathrm{H}$ NMR $\left(400 \mathrm{MHz}, \mathrm{CDCl}_{3}\right): \delta_{\mathrm{H}}=0.94,1.08\left(6 \mathrm{H}, 2 \mathrm{~s}, 2 \mathrm{CH}_{3}\right) ; 1.15(3 \mathrm{H}, \mathrm{t}$, $\left.\mathrm{CH}_{2} \mathrm{CH}_{3}, J=7.65 \mathrm{~Hz}\right) ; 2.08(1 \mathrm{H}, \mathrm{br} . \mathrm{s},-\mathrm{NH}) ; 2.13\left(2 \mathrm{H}, \mathrm{dd}, \mathrm{C}_{8}-\mathrm{H}, J=17 \mathrm{~Hz}\right) ; 2.41\left(2 \mathrm{H}, \mathrm{dd}, \mathrm{C}_{6}-\mathrm{H}, J=16.15 \mathrm{~Hz}\right)$; $3.75\left(3 \mathrm{H}, \mathrm{S},-\mathrm{OCH}_{3}\right), 4.00\left(2 \mathrm{H}, \mathrm{q},-\mathrm{CH}_{2} \mathrm{CH}_{3}, J=6.8 \mathrm{~Hz}\right) ; 4.77\left(1 \mathrm{H}, \mathrm{s}, \mathrm{C}_{4}-\mathrm{H}\right) ; 6.48\left(2 \mathrm{H}\right.$, br.s, $\left.\mathrm{NH}_{2}\right)$ and 6.76,6.83, 7.09,7.32 (4H, d,dd,d,dd Ar-H). ${ }^{13} \mathrm{C}$ NMR $\left(\mathrm{CDCl}_{3}\right): \delta_{\mathrm{C}}=14.18\left(\mathrm{CH}_{2} \underline{\mathrm{CH}}_{3}\right) ; 26.87,29.35\left(2 \mathrm{CH}_{3}\right) ; 31.61\left(\mathrm{C}_{4}\right)$; $32.15\left(\mathrm{C}_{7}\right) ; 40.74\left(\mathrm{C}_{8}\right) ; 50.73\left(\mathrm{C}_{6}\right) ; 55.20\left(-\mathrm{OCH}_{3}\right) ; 59.46\left(\underline{\mathrm{CH}}_{2} \mathrm{CH}_{3}\right) ; 78.94\left(\mathrm{C}_{3}\right) ; 110.72\left(\mathrm{C}_{4 \mathrm{a}}\right) ; 114.57\left(\mathrm{C}_{3}{ }^{\prime}\right)$; $119.82\left(\mathrm{C}_{1}^{\prime}, \mathrm{C}_{5}^{\prime}\right) ; 127.37\left(\mathrm{C}_{4}^{\prime}\right) ; 132.03\left(\mathrm{C}_{6}^{\prime}\right) ; 157.79\left(\mathrm{C}_{8 \mathrm{a}}\right) ; 158.91\left(\mathrm{C}_{2}^{\prime}\right) ; 162.10,196.70(2 \mathrm{C}=\mathrm{O})$; and 169.57 $\left(\mathrm{C}_{2}\right)$. Anal. Calcd. for $\mathrm{C}_{21} \mathrm{H}_{26} \mathrm{~N}_{2} \mathrm{O}_{4}$ (370.44): C, 68.03; H, 7.02; N, 7.56; O, 17.28. Found : C, 68.44; H, 6.99; N, 7.05; O, 17.01.

Ethyl2-amino-4-(2-hydroxyphenyl)-7,7-dimethyl-5-oxo-1,4,5,6,7,8hexahydroquinoline-3-carboxylate $\left(2_{j}\right)$

Yellow powder (1.11 gm, $87.49 \%$ yield); m.p $114{ }^{\circ} \mathrm{C}$. FTIR; 3600-2900 (br.band for $\left.-\mathrm{NH}, \mathrm{NH}_{2}, \mathrm{OH}\right)$; $1705,1655(2 \mathrm{C}=\mathrm{O})$; and $1630 \mathrm{~cm}^{-1}(\mathrm{C}=\mathrm{C}) .{ }^{1} \mathrm{H}$ NMR $\left(400 \mathrm{MHz}, \mathrm{CDCl}_{3}\right): \delta_{\mathrm{H}}=0.99\left(6 \mathrm{H}, 2 \mathrm{~s}, 2 \mathrm{CH}_{3}\right) ; 1.08(3 \mathrm{H}, \mathrm{t}$, $\left.-\mathrm{CH}_{2} \mathrm{CH}_{3}, J=7.2 \mathrm{~Hz}\right) ; 1.18(1 \mathrm{H}, \mathrm{br} . \mathrm{s},-\mathrm{NH}) ; 2.53\left(2 \mathrm{H}, \mathrm{dd}, \mathrm{C}_{8}-\mathrm{H}, J=14 \mathrm{~Hz}\right) ; 2.66$ ( 2H, s, $\left.\mathrm{C}_{6}-\mathrm{H}\right) ; 3.90$ (2H, q, $\left.\mathrm{CH}_{2} \mathrm{CH}_{3}, J=7.2 \mathrm{~Hz}\right) ; 4.67\left(1 \mathrm{H}, \mathrm{s}, \mathrm{C}_{4}-\mathrm{H}\right) ; 6.45\left(2 \mathrm{H}\right.$, br.s, $\left.\mathrm{NH}_{2}\right)$ and 7.01,7.16,7.26,7.86 (4H, dd,dd,2d, $\left.\mathrm{Ar}-\mathrm{H}\right)$. ${ }^{13} \mathrm{C}$ NMR $\left(\mathrm{CDCl}_{3}\right): \delta_{\mathrm{C}}=14.05\left(\mathrm{CH}_{2} \underline{\mathrm{CH}}_{3}\right) ; 27.21,27.78\left(2 \mathrm{CH}_{3}\right) ; 32.32\left(\mathrm{C}_{7}\right) ; 32.58\left(\mathrm{C}_{4}\right) ; 42.55\left(\mathrm{C}_{8}\right) ; 47.88\left(\mathrm{C}_{6}\right)$; $53.82\left(\underline{\mathrm{CH}}_{2} \mathrm{CH}_{3}\right) ; 99.25\left(\mathrm{C}_{3}\right) ; 115.75\left(\mathrm{C}_{3}{ }^{\prime}\right) ; 118.32\left(\mathrm{C}_{4 \mathrm{a}}\right) ; 123.38\left(\mathrm{C}_{5}{ }^{\prime}\right) ; 124.31\left(\mathrm{C}_{1}{ }^{\prime}\right) ; 127.99\left(\mathrm{C}_{4}{ }^{\prime}\right) ; 130.04\left(\mathrm{C}_{1}{ }^{1}\right)$; $147.64\left(\mathrm{C}_{8 \mathrm{a}}\right) ; 151.61\left(\mathrm{C}_{2}^{\prime}\right) ; 161.48,197.35(2 \mathrm{C}=\mathrm{O})$; and $169.73\left(\mathrm{C}_{2}\right)$. Anal. Calcd. for $\mathrm{C}_{20} \mathrm{H}_{24} \mathrm{~N}_{2} \mathrm{O}_{4}(356.42)$ : $\mathrm{C}$, 67.34; H, 6.73; N, 7.86; O, 17.96. Found: C, 67.82; H, 6.37; N, 7.42; O, 17.55.

\section{Results and Discussion}

\subsection{Catalytic Test}

The synthesis of quinoline derivatives via four-component one-pot reaction of dimedone, pchlorobenzaldehyde, ethyl cyanoacetate and ammonium acetate is represented in scheme 1 . Neat reaction (absence of catalyst) showed $\sim 25 \%$ yield in $6 \mathrm{~h}$ using ethanol under refluxing condition. The neat reaction carried out under ultrasonic irradiation resulted in poor isolated yield of the product ( 29\%) in 15 (min). In order to find out suitable catalyst, for the synthesis of quinoline derivatives via MCR under ultrasound irradiation, two different catalysts (CS/CuNPs and CS/CuNPs/TPP) were synthesized and tested. 


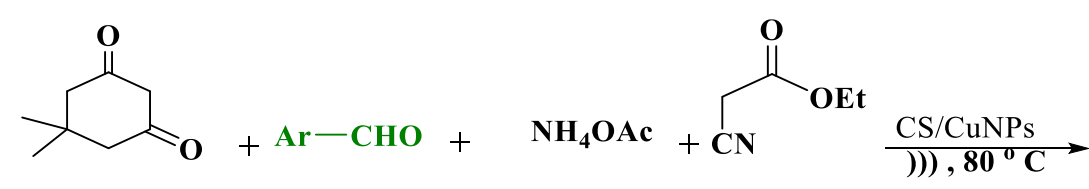

$\mathbf{1} \mathbf{a}-\mathbf{j}$<smiles>CCOC(=O)C1=C(N)NC2=C(C(=O)CC(C)(C)C2)C1[Al]</smiles>

2 a-j

Scheme 1: Synthesis protocol of quinolone derivatives.

The effect of mass of the two catalysts was studied and the results displayed in Figure 1. The results clearly showed that $0.1 \mathrm{~g}$ of CS/CuNPs provided high product's yield $(\sim 90 \%)$ relative to the other catalyst under the same reaction conditions.

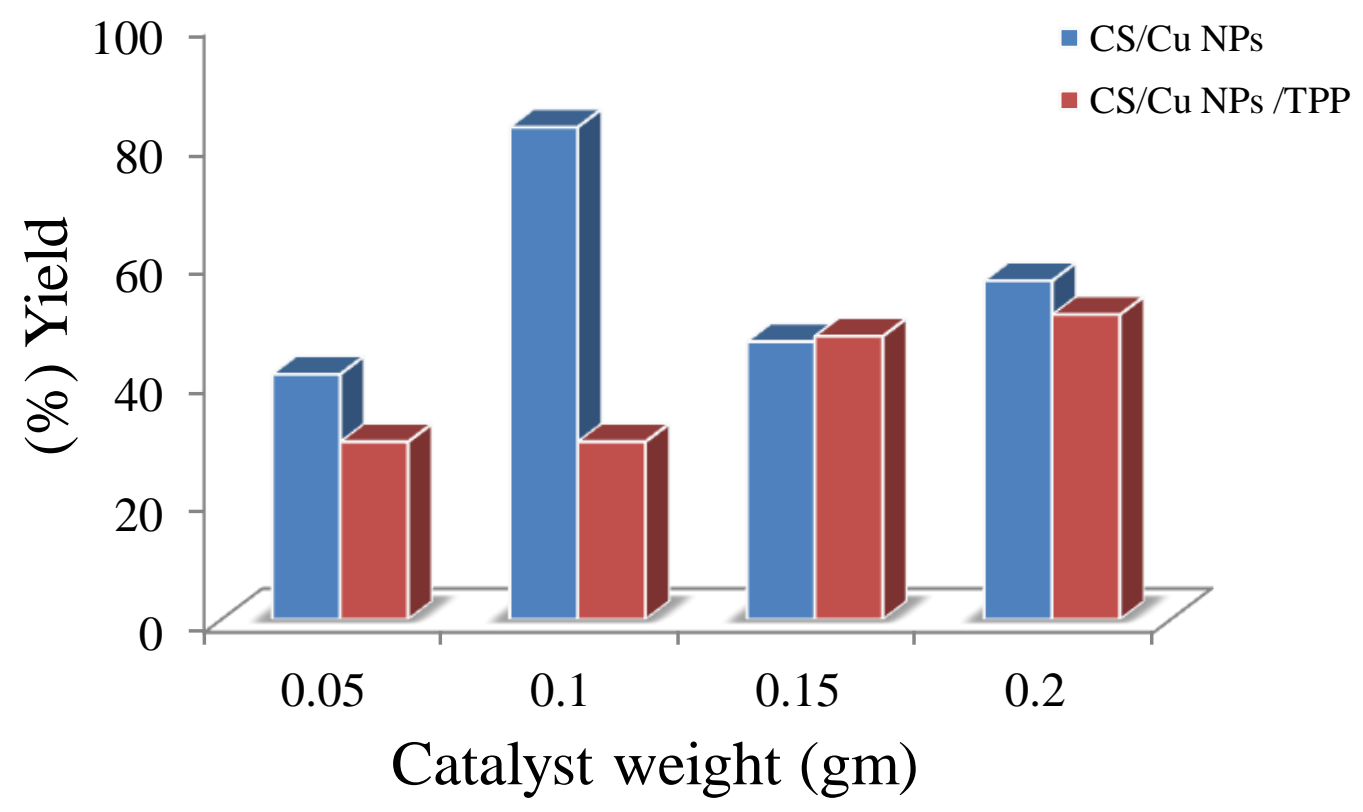

Figure 1. Catalytic activity of all the investigated catalysts with different masses*

*Reaction conditions: dimedone $(3.6 \mathrm{mmol})$, aromatic aldehyde $(3.6 \mathrm{mmol})$, ethyl cyanoacetoacetate (3.6 mmol), ammonium acetate $(28.8 \mathrm{mmol})$ and different weight of catalysts in $10 \mathrm{ml}$ absolute ethanol under ultrasonic irradiation at $80^{\circ} \mathrm{C}$ for $15 \mathrm{~min}$.

The ultrasonic irradiation method was selected based on the advantages of this method in comparison to the conventional one in enhancing the product yield in shorter reaction time. Therefore, a variety of quinolines derivatives were also synthesized via this method by using the highly efficient catalyst (CS/CuNPs, $0.1 \mathrm{~g})$. The optimal reaction conditions were determined to be catalyst loading: $0.1 \mathrm{~g} \mathrm{CS} / \mathrm{CuNPs}$; dimedone (3.6 mmol); aromatic aldehyde $(3.6 \mathrm{mmol})$; ethylcyanoacetoacetate $(3.6 \mathrm{mmol})$; ammonium acetate $(28.8 \mathrm{mmol})$ and $10 \mathrm{ml}$ absolute ethanol under ultrasonic irradiation at $80^{\circ} \mathrm{C}$ for $15 \mathrm{~min}$. Then, with the optimum reaction parameters, the catalytic performance of the catalyst was examined in MCR for quinoline derivatives and results are displayed in Table 1. 
Table 1: Effect of CS/CuNPs catalyst on the synthesis of polyhydroquinolines $2^{a-j}$ using various aromatic aldehydes**

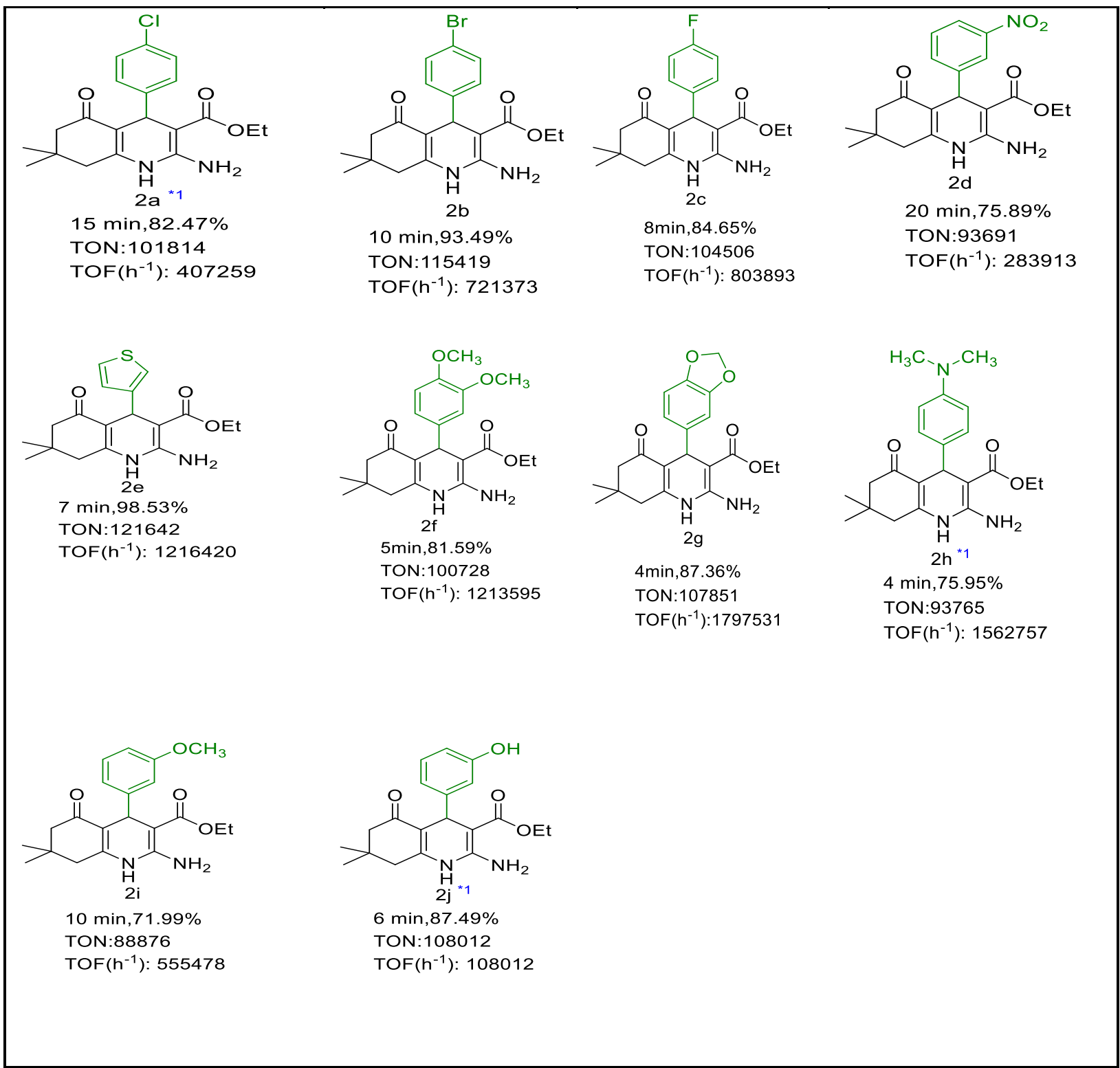

${ }^{* 1}[36] * *$ Reaction conditions: dimedone $(3.6 \mathrm{mmol})$, aromatic aldehyde $(3.6 \mathrm{mmol})$, ethylcyanoacetoacetate $(3.6 \mathrm{mmol})$, ammoniumacetate $(28.8 \mathrm{mmol})$ and $0.1 \mathrm{~g}$ of catalyst in $10 \mathrm{ml}$ absolute ethanol under ultrasonic irradiation at $80^{\circ} \mathrm{C}$ for 15 min.; TON: (turnover number, yield of product/ per mol of $\mathrm{Cu}$ ); TOF: (turn over frequency, TON/time of reaction).

As seen from the data in Table 1 catalyst provides an efficient synthesis of a new quinoline derivatives with high yield productivity, in short reaction time. In addition, the TON and TOF values were calculated and are 
existing in Table 1. A remarkably high TON and TOF values were found with small mass of catalyst. Considering such cyclocondensation reactions, chitosan decorated copper nanoparticles is considered to be a suitable efficient catalyst. Basically, catalytic activity of nanoparticles is related to the size of the particles and the good dispersion of active species on the catalyst's support. Therefore, extensive characterization of the most efficient CS/CuNPs catalyst was attained and presented in the next section.

Many catalysts have been used for one pot-catalytic synthesis of organic precursors utilizing nanocrystalline and nanoparticle catalysts such as $\mathrm{ClO}_{4} / \mathrm{Zr}-\mathrm{MCM}-41$ nanoparticles [37], $\mathrm{Fe}_{3} \mathrm{O}_{4} @ \mathrm{~B}-\mathrm{MCM}-41$ [38], and $\mathrm{ZnO}$ nanoparticles [39]. All of these catalysts showed pronounced catalytic activity due to their nanosized and large surface area features. In contrary to that the produced $\%$ yield of products especially after re-use of catalysts for four time was not sufficient relative to our proposed catalyst in the present work. In order to study the sustainability of the present efficient catalyst towards four components one-pot catalytic synthesis of novel quinoline derivatives under ultrasound irradiation, the re-use test was carried out and the results are given in the following section.

\subsection{Reusability Procedure}

Reusability is crucial for supported catalysts [40]. Therefore, the same catalytic particles were used after filtration from the reaction mixture for several reactions under the same conditions. Typically, after $15 \mathrm{~min}$ ultrasound irradiation, CS/CuNPs was filtered and washed 4-6 times with hot ethanol to remove all the unreacted educts then dried at room temperature for $24 \mathrm{~h}$. The dried catalyst was used in the subsequent runs and the results are displayed in Figure 2.

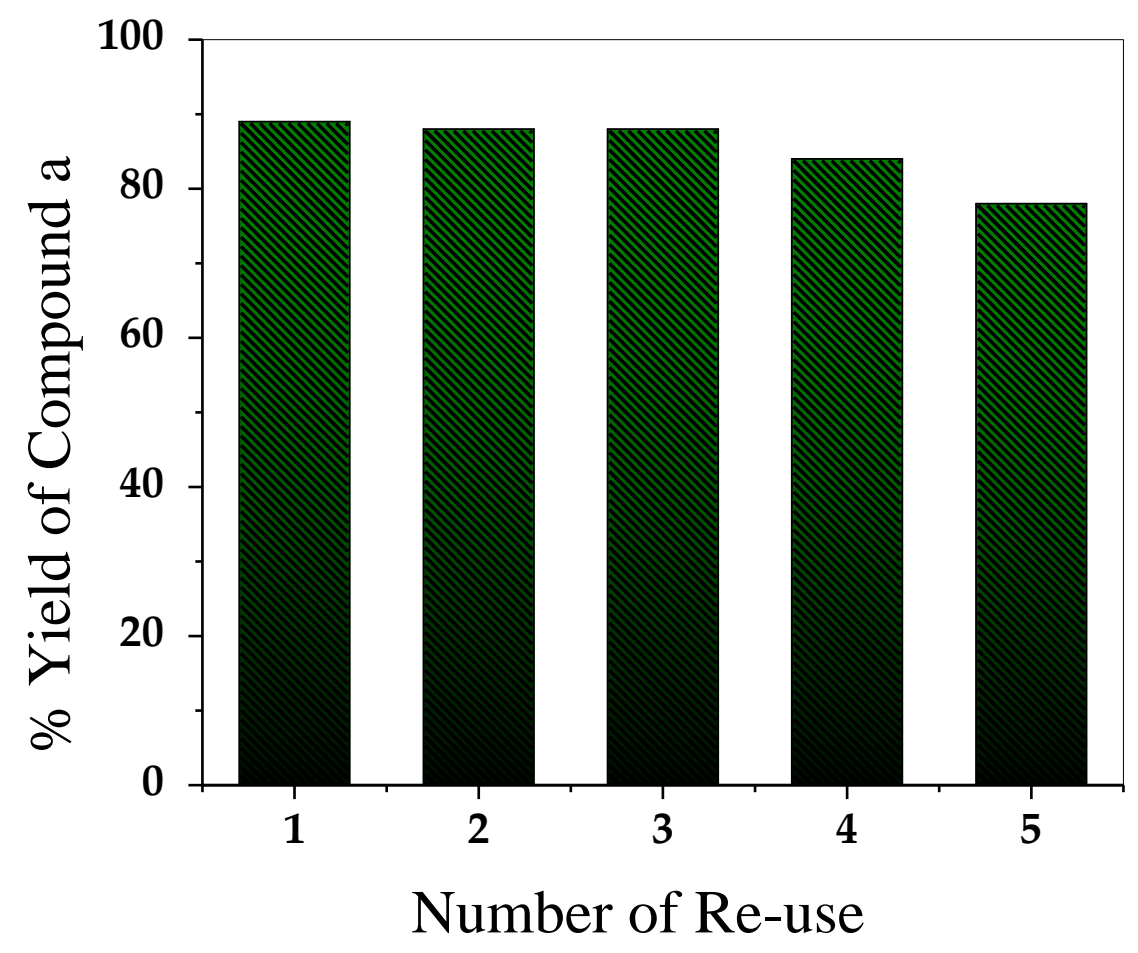

Figure 2. Robust feature of CS/CuNPs catalyst after five times r-use.

The results shown in Figure 2 depicted that there is no apparent decay of catalytic activity even after 5 runs, and the attained yields of the reusability test are within the experimental deviations. 


\subsection{Catalyst Characterization 3.3.1 FTIR}

FT-IR spectra of chitosan and CS/CuNPs Figure 3 displayed a broad band for $\mathrm{OH}$ and NH stretching of amine groups located at $3250 \mathrm{~cm}^{-1}$. The existence of band at $1553 \mathrm{~cm}^{-1}$ is due to the presence of the $\mathrm{NH}_{2}$ groups. Stretching vibrations due to $\mathrm{C}-\mathrm{OH}$ and $\mathrm{C}-\mathrm{N}$ appeared consequently in the absorption bands in the range 1016 and $1402 \mathrm{~cm}^{-1}$. The absorption band placed at $2936 \mathrm{~cm}^{-1}$ is credited to the $\mathrm{C}-\mathrm{H}$ stretching mode of methylene groups[41]. The decoration of chitosan by copper nanoparticles resulted in the formation of new intense peaks in the fingerprint region at low -frequency $\left(600-500 \mathrm{~cm}^{-1}\right)$ due to formation of $\mathrm{Cu}-\mathrm{N}$ and $\mathrm{Cu}-\mathrm{O}$ coordinate bonds. Furthermore, the peak at $612 \mathrm{~cm}^{-1}$ assigns for CuNPs-chitosan interaction, indication that NPs were capped by the biopolymer [34].

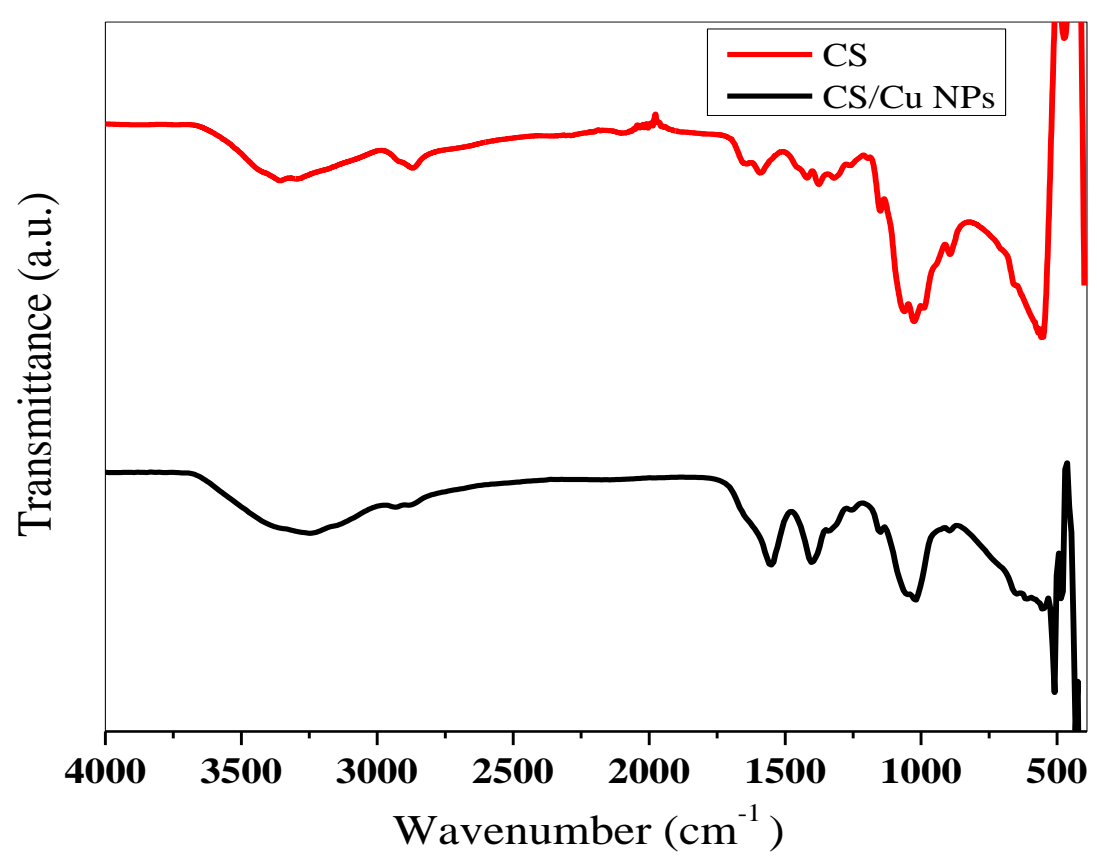

Figure 3. FTIR spectra of pure chitosan and CS/CuNPs catalyst.

\subsubsection{TGA analyses}

Thermograms of CS and CS/CuNPs samples are shown in Figure 4. The TGA study is used to illuminate the thermal stability and approach of decomposition of the pristine chitosan and copper decorated chitosan. The TGA thermogram of investigated samples display three mass loss stages. The preliminary mass loss (8\%) for $\mathrm{CS}$ and $(21 \%)$ for $\mathrm{CS} / \mathrm{CuNPs}$ in the temperature range $25-200^{\circ} \mathrm{C}$, could be to attributed to the hygroscopic nature of the chitosan and dehydration of copper decorated chitosan sample [42]. The second thermal stage in the temperature range $200-400{ }^{\circ} \mathrm{C}$ displayed 48 and $40 \%$ mass loss for CS and CS/CuNPs, respectively is mainly assigned to de-polymerization along with breakdown of acetylated and deacetylated unit of chitosan. The third thermal decomposition stage $\left(400-550^{\circ} \mathrm{C}\right)$ was accompanied by about $30 \%$ mass loss for both investigated samples. The total mass loss of copper nanoparticles decorated chitosan is higher than that of pristine chitosan ( $10 \%$ higher) as the introduction of a metal nanoparticles into the biopolymer matrix, affect the chain packing and causes releasing of the packed assembly $[43,44]$. 


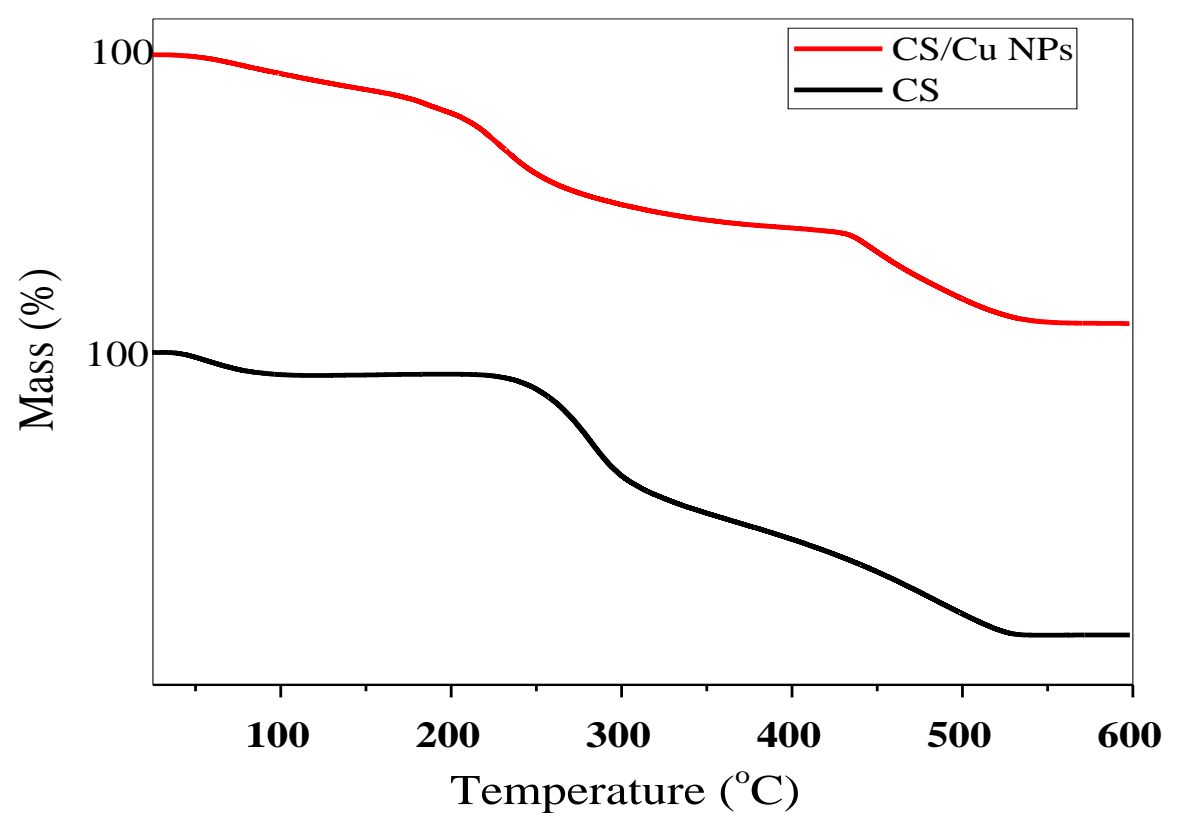

Figure 4. TGA of chitosan (CS) and CS/CuNPs.

\subsubsection{XRD analyses}

X-ray diffraction patterns of pure chitosan (CS) and chitosan decorated copper nanoparticles (CS/CuNPs) are displayed in Figure 5. Some characteristic peaks for chitosan at $2 \theta=11.5^{\circ}$ and $22.5^{\circ}$ were observed [45,46]. A slight right shift with wider peak at $2 \theta=23^{\circ}$ recommends the decrease in crystallinity after anchoring copper nanoparticles in the chitosan structure. The main structure of chitosan was not disturbed with the absence of any characteristic peaks for copper nanoparticles. This observation suggests the dispersion of copper nanoparticles over the surface of chitosan and the structure of chitosan was not changed during the preparation method.

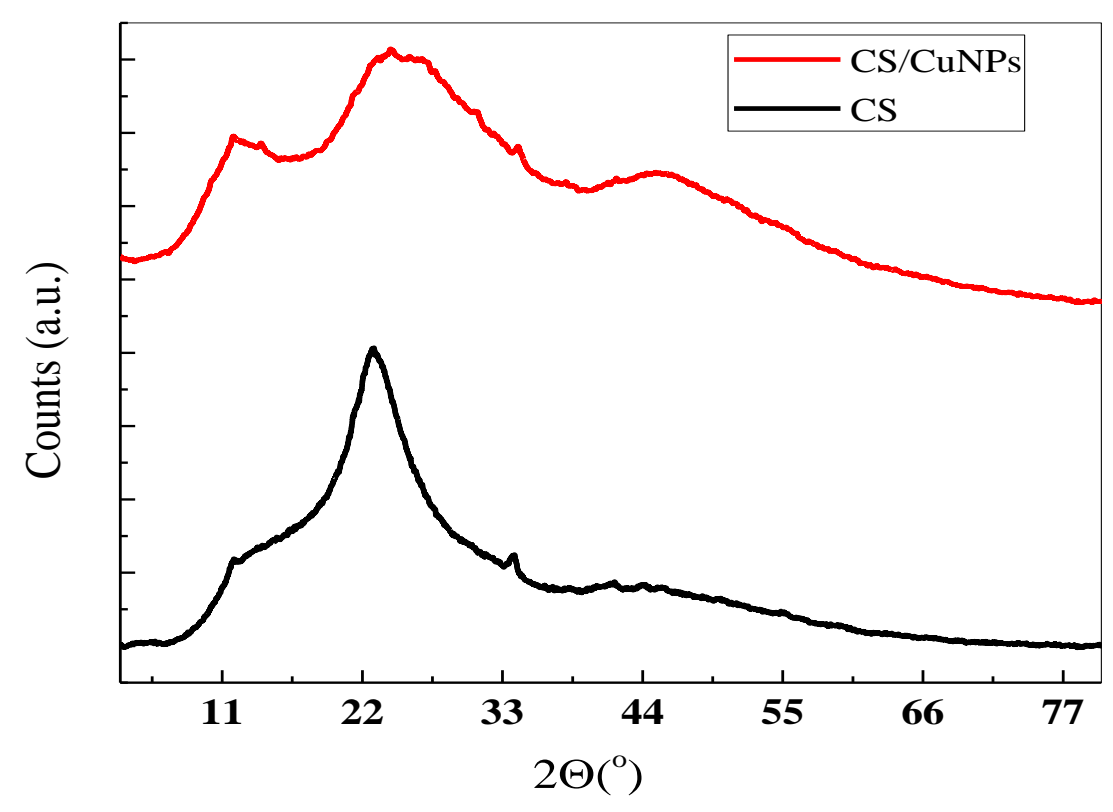

Figure 5. XRD patterns of chitosan (CS) and CS/CuNPs. 


\subsubsection{SEM-EDX}

The morphology of chitosan decorated copper nanoparticles sample (CuNPs) described by SEM image Figure 6 displayed asymmetrical deposits of chitosan. The nonattendance of copper nanoparticles could be ascribed to the good scattering of copper nanoparticles over chitosan. EDX spectra Figure 7 showed copper in addition to carbon, nitrogen and oxygen elements. The atomic \% of copper should be complemented by XPS analysis in order to give accurate turnover number of copper relative to the total atomic percentage derived from ICP-AES analysis.

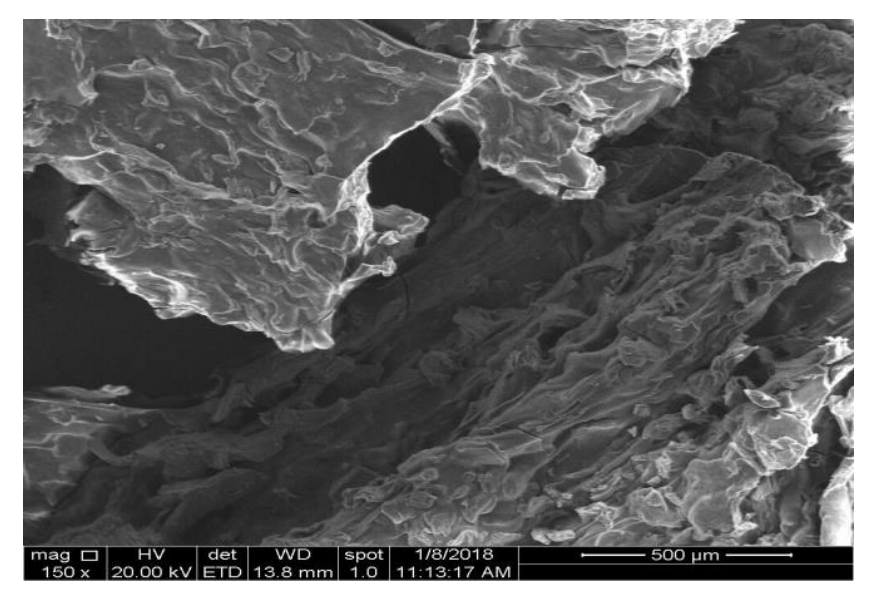

Figure 6. SEM image for CS/CuNPs.

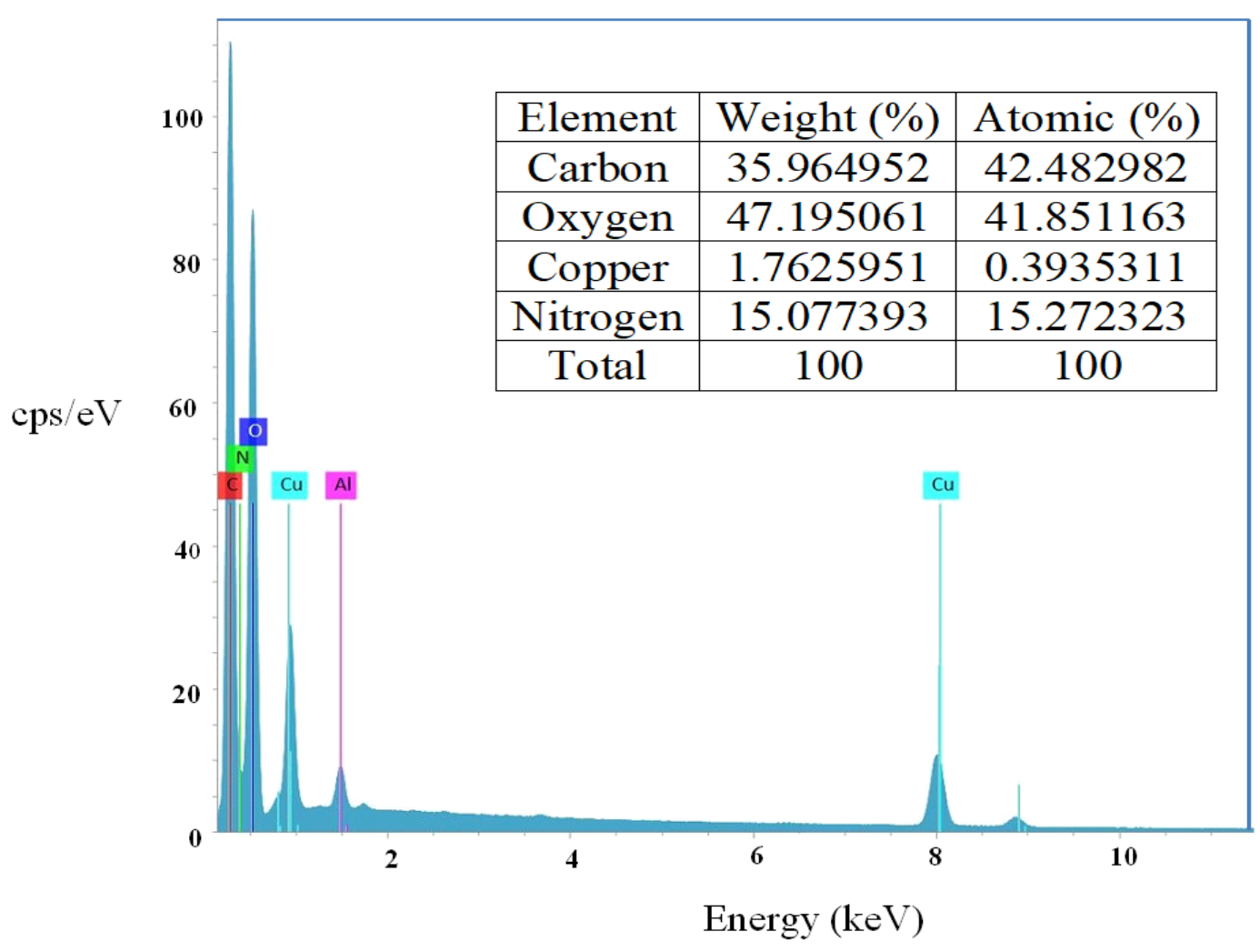

Figure 7. EDX spectra of Chitosan decorated copper nanoparticles. 


\subsubsection{XPS and HRTEM}

XPS peaks at 932.7 and $952.5 \mathrm{eV}$ corresponding to $\mathrm{Cu} 2 \mathrm{p} 3 / 2$ and $\mathrm{Cu} 2 \mathrm{p} 1 / 2$, respectively, which confirmed the presence of copper, which is not appeared in XRD patterns. Copper nanoparticles are fashioned in three diverse oxidation states, which could improve the catalytic efficacy of the synthesized catalyst. The number of moles of copper nanoparticles was detected from both EDX and XPS analyses for the determination of turnover number of active species. TEM images (Figure 8 right-side) show copper nanoparticles are well dispersed over chitosan. The corresponding selected area diffraction (SAED pattern) shows uniform distribution of copper NPs in two faces (111) and (110). These information about the uniform distribution of copper species with different oxidation states in different crystallographic faces could deliver elucidation of the superior catalytic activity of the catalyst and the suitability of the catalyst's support in avoiding the agglomeration of copper nanoparticles.
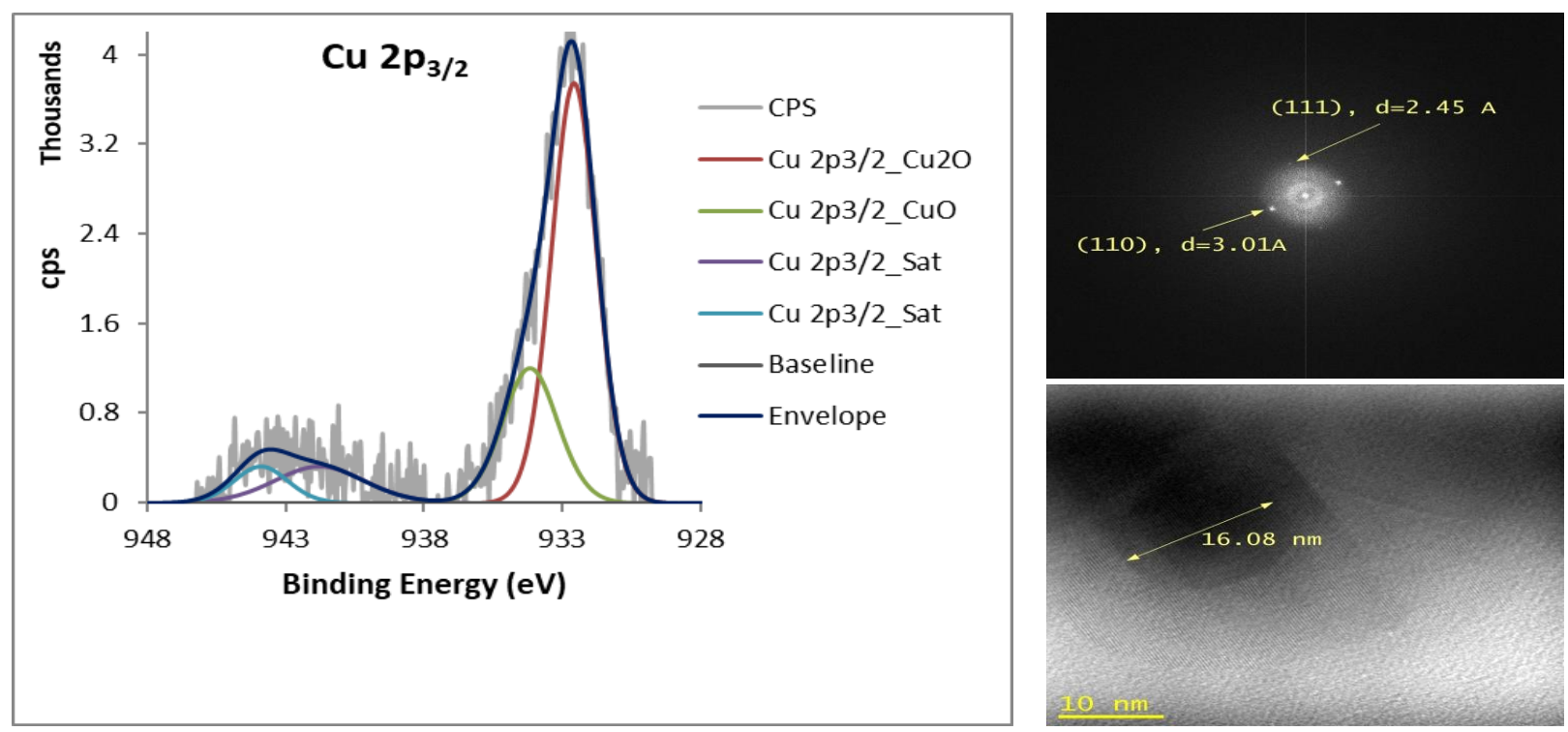

Figure 8. XPS (left-side) and HRTEM images (right-side) for CS/CuNPs

\section{Tentative Mechanism}

A tentative mechanism for multicomponent reaction of quinolines derivatives over $\mathrm{CS} / \mathrm{CuNPs}$ has been proposed to occur via three different reaction steps (Scheme 2). Firstly, the well dispersed copper nanoparticles facilitate the electrophilicity of carbonyl group of the aldehyde via reduction of $\mathrm{Cu}^{2+}$ ions into $\mathrm{Cu}^{\circ}$, which resulted in ease of attack on the active methylene carbon of ethylcyanoacetate and elimination of water. Secondly, the reaction proceeded via Michael addition assisted by basic sites of the catalyst then followed by the last step in ionic mechanism. 


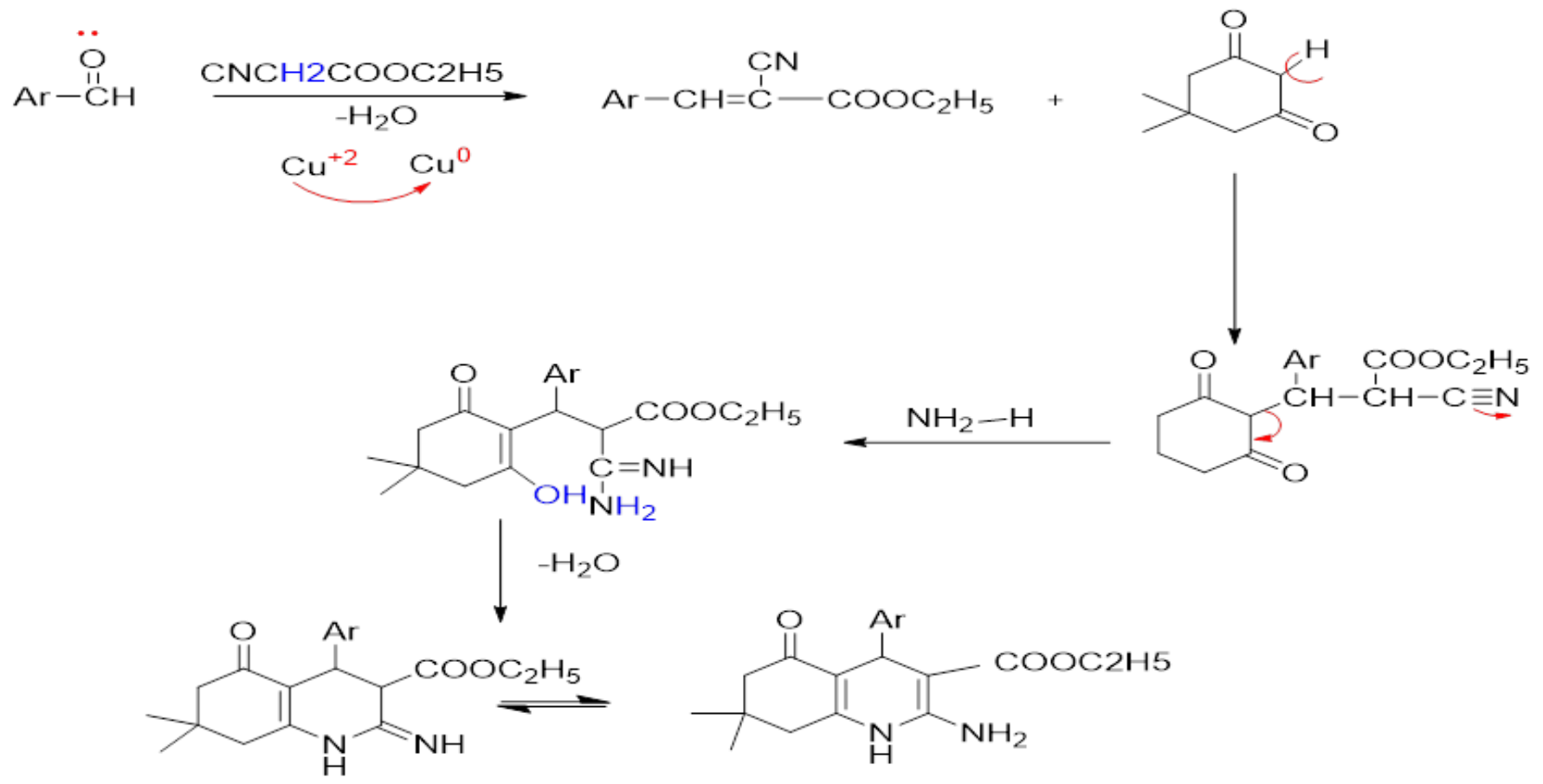

Scheme 2: proposed mechanism for the synthesis of quinolone derivatives.

\section{Conclusions}

A series of chitosan decorated copper nanoparticles were successfully synthesized using green methods. All the investigated catalysts showed high catalytic performance towards the synthesis, in acceptable yield, of novel quinoline derivatives at very short time under ultrasonic irradiation. CS/CuNPs catalyst showed superior TOF for the synthesis of novel quinoline derivatives of expected biological activity. Thanks to the well dispersion of nanoparticles over natural inactive good support the pronounced superior catalytic efficacy was attained. The re-use of this particular catalyst for five times without appreciable change in its catalytic efficacy open the gate towards promising noble metal free catalysts for fine chemical products utilizing green protocol.

\section{Acknowledgments}

K.S.A. acknowledges chemistry department, faculty of science, King Abdulaziz University for providing the lab facilities for doing the research during her MSc. Thesis.

\section{References and Notes}

[1] Kwiek, J. J., Haystead, T. A., Rudolph, J., 2004. Kinetic mechanism of quinone oxidoreductase 2 and its inhibition by the antimalarial quinolines. Biochemistry, 43(15), pp.4538-4547.

[2] Kogn, L. K., Pavam, C., Veronese, D., Coelho, F., De, C. J., Almeida, W. P., 2006. Antiproliferative effect of Baylis-Hillman adducts and a new phthalide derivative on human tumor cell lines. European Journal of Medicinal Chemistry, 41(6), pp.738-744.

[3] Ahmed, N., Badahdah, K., Qassar, H., 2017. Novel quinoline bearing sulfonamide derivatives and their cytotoxic activity against MCF7 cell line. Medicinal Chemistry Research, 26(6), pp.1201-1212.

[4] Roma, G., Di, B. M., Grossi, G., Mattioli, F., Ghia, M., 2000. 1, 8-Naphthyridines IV. 9substituted N, N-dialkyl-5-(alkylamino or cycloalkylamino)[1, 2, 4] triazolo [4, 3-a][1, 8] naphthyridine-6-carboxamides, new compounds with anti-aggressive and potent antiinflammatory activities. European Journal of Medicinal Chemistry, 35 (11), pp.1021-1035.

[5] Bekhit, A. A., El-sayed, O. A., Aboulmagd, E., Park, J. Y., 2004. Tetrazolo [1, 5-a] quinoline as a potential promising new scaffold for the synthesis of novel anti-inflammatory and antibacterial agents. European Journal of Medicinal Chemistry, 39(3), pp.249-255. 
[6] Dube, D., Blouin, M., Brideau, C., Chan, C. C., Desmarais, S., Ethier, D., Falgueyret, J. P., Friesen, R. W., Girard, M., Girard, Y., 1998. Quinolines as potent 5-lipoxygenase inhibitors: synthesis and biological profile of L-746,530. Bioorganic \& Medicinal Chemistry Letters, 11(8), pp1255-1260.

[7] Ko, T. C., Hour, M. J., Lien, J. C., Teng, C. M., Lee, K, H., Kuo, S. C., Huang, L. J., 2001. Synthesis of 4-alkoxy-2-phenylquinoline derivatives as potent antiplatelet agents. Bioorganic \& Medicinal Chemistry Letters, 11(3), pp.279-282.

[8] Murganantham, N., Sivkumar, R., Anbalagan, N., Gunaskaran,V., Leonard, J. T., 2004. Synthesis, anticonvulsant and antihypertensive activities of 8-substituted quinoline derivatives. Biological and Pharmaceutical Bulletin, 27(10), pp.1683-1687.

[9] Das, B., Ravikanth, B., Ramu, R., Rao, B. V., 2006. An efficient one-pot synthesis of polyhydroquinolines at room temperature using HY-zeolite. Chemical and Pharmaceutical Bulletin, 54(7), pp.1044-1045.

[10] Sapkal, S. B., Shelke, K. F., Shingate, B. B., Shingare, M. S., 2009. Nickel nanoparticlecatalyzed facile and efficient one-pot synthesis of polyhydroquinoline derivatives via Hantzsch condensation under solvent-free conditions. Tetrahedron Letters, 50(15), pp.1754-1756.

[11] Dodoni, A., Massi, A., Minghini, E., Bertolasi, V., 2004. Multicomponent Hantzsch cyclocondensation as a route to highly functionalized 2-and 4-dihydropyridylalanines, 2-and 4pyridylalanines, and their $\mathrm{N}$-oxides: preparation via a polymer-assisted solution-phase approach. Tetrahedron, 60(10), pp.2311-2326.

[12] Karade, N. N., Budhewar, V. H., Shinse, S. V., Jadhav, W. N., 2007. L-proline as an efficient organo-catalyst for the synthesis of polyhydroquinoline via multicomponent Hantzsch reaction. Letters in Organic Chemistry, 4(1), pp.16-19.

[13] Pilli, S., Bhunia, P., Yan, S., Leblanc, R., Tyagi, R., Surampalli, R., 2011. Ultrasonic pretreatment of sludge: a review. Ultrasonics Sonochemistry, 18(1), pp.1-18.

[14] Hussain, S., Jadhav, S., Rai, M., Farooqui, M., 2014. One-pot neda catalyzed Knovenagel condensation under ultrasonic irradiation in solvent-free medium. International Journal of Pharmaceutical, Chemical \& Biological Sciences, 4(1), pp.126-128.

[15] Polshettiwar, V., Varma, R. S., 2010. Green chemistry by nano-catalysis. Green Chemistry, 12(5), pp.743-754.

[16] Rostamizadeh, S., Shadjou, N., Azad, M., Jalali, N., 2012. ( $\left.\alpha-\mathrm{Fe}_{2} \mathrm{O}_{3}\right)-\mathrm{MCM}-41$ as a magnetically recoverable nanocatalyst for the synthesis of pyrazolo [4, 3-c] pyridines at room temperature. Catalysis Communications, 26(9), pp.218-224.

[17] Mokhtar, M., Saleh, T., Ahmed, N., Al-Thabaiti, S., Al-Shereef, R., 2011. An eco-friendly Nsulfonylation of amines using stable and reusable $\mathrm{Zn}-\mathrm{Al}-$ hydrotalcite solid base catalyst under ultrasound irradiation. Ultrasonics Sonochemistry, 18(1), pp.172-176.

[18] Mokhtar, M., Saleh, T., Basahel, S. N., 2012. Mg-Al hydrotalcites as efficient catalysts for azaMichael addition reaction: a green protocol. Journal of Molecular Catalysis A: Chemical, 353(2), pp. 122-131.

[19] Saleh, T. S., Narasimharao, K., Ahmed, N. S., Basahel, S. N., Al-Thabaiti, S., Mokhtar, M., 2013. Mg-Al hydrotalcite as an efficient catalyst for microwave assisted regioselective 1, 3dipolar cycloaddition of nitrilimines with the enaminone derivatives: A green protocol. Journal of Molecular Catalysis A: Chemical, 367(2), pp.12-22.

[20] Narasimharao, K., Al-Sabban, E., Saleh, T., Gallastegui, G. A., Sanfiz, C. A., Basahel, S. N., Al-Thabaiti, S., Alyoubi, A., Obaid, A., Mokhtar, M., 2013. Microwave assisted efficient protocol for the classic Ullmann homocoupling reaction using $\mathrm{Cu}-\mathrm{Mg}-\mathrm{Al}$ hydrotalcite catalysts. Journal of Molecular Catalysis A: Chemical, 379(11), pp.152-162.

[21] Bagabas, A. A., Mokhtar, M., Akhmedov, V. M., Narasimharao, K., Basahel, S. N., Al-Rabiah, A., 2014. Ru-C-ZnO composite catalysts for the cynthesis of methyl isobutyl ketone via single step gas phase acetone self-condensation. Catalysis Letters, 144(7), pp.1278-1288. 
[22] Basahel, S. N., Ahmed, N., Narasimharao, K., Mokhtar, M., 2016. Simple and efficient protocol for synthesis of pyrido [1, 2-a] pyrimidin-4-one derivatives over solid heteropolyacid catalysts. RSC Advances, 6(15), pp.11921-11932.

[23] Koga, H., Tokunaga, E., Hidaka, M., Umrmura, Y., Saito, T., Isogal, A., Kitaoka, T., 2010. Topochemical synthesis and catalysis of metal nanoparticles exposed on crystalline cellulose nanofibers. Chemical Communications, 46(45), pp.8567-8569.

[24] Wang, X., Hu, P., Xue, F., Wei, Y., 2014. Cellulose-supported N-heterocyclic carbenepalladium catalyst: synthesis and its applications in the Suzuki cross-coupling reaction. Carbohydrate Polymers, 114(9), pp.476-483.

[25] Rinudo, M., 2006. Chitin and chitosan: properties and applications. Progress in Polymer Science, 31(7), pp.603-632.

[26] Baig, R. N., Varma, R. S., 2013. Copper on chitosan: a recyclable heterogeneous catalyst for azide-alkyne cycloaddition reactions in water. Green Chemistry, 15(7), pp.1839-1843.

[27] Yang, B., Mao, Z., Zhu, X., Wan, Y. 2015. Functionalised chitosan as a green, recyclable, supported catalyst for the copper-catalysed Ullmann CN coupling reaction in water. Catalysis Communications, 60(2), pp.92-95.

[28] Baig, R. N., Nadagouda, M. N., Varma, R. S., 2014. Ruthenium on chitosan: a recyclable heterogeneous catalyst for aqueous hydration of nitriles to amides. Green Chemistry, 16(4), pp.2122-2127.

[29] Yan, K., Chen, A., 2014. Selective hydrogenation of furfural and levulinic acid to biofuels on the ecofriendly Cu-Fe catalyst. Fuel, 115(1), pp.101-108.

[30] Hardy, J. J., Hubert, S., Macquarrie, D. J., Wilson, A. J., 2004. Chitosan-based heterogeneous catalysts for Suzuki and Heck reactions. Green Chemistry, 6(1), pp. 53-56.

[31] Qiu, Y., Ma, Z., Hu, P., 2014. Environmentally benign magnetic chitosan $/ \mathrm{Fe}_{2} \mathrm{O}_{3}$ composites as reductant and stabilizer for anchoring $\mathrm{Au}$ NPs and their catalytic reduction of 4-nitrophenol. Journal of Materials Chemistry A, 2(33), pp.13471-13478.

[32] Zhou, J., Dong, Z., Yang, H., Shi, Z., Zhou, X., Li, R., 2013. Pd immobilized on magnetic chitosan as a heterogeneous catalyst for acetalization and hydrogenation reactions. Applied Surface Science, 279(8), pp.360-366.

[33] Frindy, S., Elkadib, A., Lahcini, M., Primo, A., Garcia, H., 2015. Copper nanoparticles stabilized in a porous chitosan aerogel as a heterogeneous catalyst for $\mathrm{C}-\mathrm{S}$ cross-coupling. ChemCatChem, 7(20), pp.3307-3315.

[34] Manikandan, A., Sathiyabama, M., 2015. Green synthesis of copper-chitosan nanoparticles and study of its antibacterial activity. Journal of Nanomedicine \& Nanotechnology, 6(1), pp.1-5.

[35] Huang, H., Yang, X., 2004. Synthesis of chitosan-stabilized gold nanoparticles in the absence/presence of tripolyphosphate. Biomacromolecules, 5(9), pp.2340-2346.

[36] Kumar, S., Sharma, P., Kapoor, K.K., Hundal, M.S., 2008.An efficient, catalyst-and solventfree, four-component, and one-pot synthesis of polyhydroquinolines on grinding. Tetrahedron 64, pp.536-542.

[37] Abdollahi, A., M., Hoseinkhan, S., 2016. ClO $4 / Z r-M C M-41$ nanoparticles prepared at mild conditions: a novel solid acid catalyst for the synthesis of polyhydroquinolines. Journal of the Iranian Chemical Society, 13(7), pp.1339-1347.

[38] Abdollahi, A., M., Rezaeipoor, A., 2016. Fe $\mathrm{O}_{4} @$ B-MCM-41: a new magnetically recoverable nanostructured catalyst for the synthesis of polyhydroquinolines. Journal of Magnetism and Magnetic Materials, 398(1), pp.205-214.

[39] Kassaee, M., Masrouri, H., Movahedi, F., 2010. ZnO-nanoparticle-promoted synthesis of polyhydroquinoline derivatives via multicomponent Hantzsch reaction. Monatshefte für ChemieChemical Monthly, 141(12), pp.317-322.

[40] Webb, J., D., Macquarrie, S., Mceleney, K., Crudden, C., M., 2007. Mesoporous silicasupported $\mathrm{Pd}$ catalysts: an investigation into structure, activity, leaching and heterogeneity. 
Journal of Catalysis, 252(11), pp.97-109.

[41] Sudheesh, N., Sharma, S., K., Shukla, R., S., 2010. Chitosan as an eco-friendly solid base catalyst for the solvent-free synthesis of jasminaldehyde. Journal of Molecular Catalysis A: Chemical, 321(1-2), pp.77-82.

[42] Kumari, S., Pathak, D., D., 2015. Synthesis and development of chitosan anchored copper (II) Schiff base complexes as heterogeneous catalysts for $\mathrm{N}$-arylation of amines. Tetrahedron Letters, 56(27), pp. 4135-4142.

[43] Antony, R., David, S., T., Saravanan, K., Karuppasamy, K., Balakumar, S., 2013. Synthesis, spectrochemical characterisation and catalytic activity of transition metal complexes derived from Schiff base modified chitosan. Spectrochimica Acta Part A: Molecular and Biomolecular Spectroscopy, 103(2), pp. 423-430.

[44] Tirkistani, F. A., 1998. Thermal analysis of some chitosan Schiff bases. Polymer Degradation and Stability, 60(1), pp.67-70.

[45] Kumar, S., Dutta, P., Koh, J., 2011. A physico-chemical and biological study of novel chitosanchloroquinoline derivative for biomedical applications. International Journal of Biological Macromolecules, 49(3), pp.356-361.

[46] Roberts, G. A., 1992. Chitin Chemistry. Macmillan International Higher Education. 